\title{
金属有机骨架的高通量计算節选研究进展
}

\author{
刘治鲁 $\dagger, a, b$ 李炜 ${ }^{\dagger}, a, b$ 刘昊 ${ }^{b}$ 庄旭东 ${ }^{b}$ 李松 $*, a, b$ \\ ( ${ }^{a}$ 华中科技大学 中欧清洁与可再生能源学院 武汉 430074) \\ ( $b$ 华中科技大学 能源与动力工程学院煤燃烧国家重点实验室 武汉 430074)
}

\begin{abstract}
摘要 近年来, 金属有机骨架(Metal-Organic Frameworks, MOFs)在气体吸附分离领域的研究获得爆发式增长. 随着 MOFs 数量的剧增, 高通量计算笁选成为从大量 MOFs 中发现高性能目标材料和挖掘其构效关系的最有效研究方法. 本综述对 MOFs 的高通量计算筛选中所用到的数据库包括实验合成的 MOFs 组成的数据库 (experimental MOFs, eMOFs)和计算机设计的 MOFs 数据库(hypothetical MOFs, hMOFs)、计算篮选方法包括基于分子模拟和机器学习的篮 选方法, 及其在 $\mathrm{CH}_{4}$ 储存、 $\mathrm{H}_{2}$ 储存、 $\mathrm{CO}_{2}$ 捕捉和其他气体分离领域的研究进展进行了总结. 旨在通过梳理该领域的研 究进展和思路, 明确未来的研究方向和面临的挑战, 加快 MOFs 的研发进程, 促进 MOFs 的商业化应用.
\end{abstract}

关键词 金属有机骨架; 高通量篮选; 分子模拟; 机器学习; 吸附分离

\section{Research Progress of High-throughput Computational Screening of Metal-Organic Frameworks}
Liu, Zhilu ${ }^{\dagger}, a, b$
$\mathrm{Li}, \mathrm{Wei}^{\dagger}, a, b$
Liu, $\mathrm{Hao}^{b}$
Zhuang, Xudong ${ }^{b}$
$\mathrm{Li}$, Song ${ }^{*, a, b}$

$\left({ }^{a}\right.$ China-EU Institute for Clean and Renewable Energy, Huazhong University of Science and Technology, Wuhan 430074) $\left({ }^{b}\right.$ State Key Laboratory of Coal Combustion, School of Energy and Power Engineering, Huazhong University of Science and Technology, Wuhan 430074)

\begin{abstract}
During the past decades, extensive investigations on metal-organic frameworks (MOFs) with ultrahigh surface area for gas adsorption and separation have been reported. With the increasing number of possible MOFs, it has been a great challenge to discover high-performing MOFs of interest from numerous structures. High-throughput computational screening (HTCS) is a powerful tool to accelerate the development of MOFs for application of interest and explores the quantitative structure-property relationship (QSPR) to facilitate the rational design of top-performing MOFs. In this review, we summarize the MOF databases used for HTCS, mainly including MOFs collected from experimentally synthesized MOFs (i.e. eMOFs), and the hypothetical MOFs constructed by computer-aided tools (i.e. hMOFs). Moreover, there are currently two important screening strategies, molecular simulation and machine learning-based HTCS. A vast majority of HTCS have been performed by molecular simulations including grand canonical Monte Carlo (GCMC) and molecular dynamics (MD) simulations, in which the accuracy of force field parameters play a criticl role in the reliability of HTCS. GCMC is able to predict the adsorption performance of MOFs such as adsorption capacity, selectivity and heat of adsorption, whereas MD is commonly used to estimate the dynamic property of adsorbates, e.g. diffusion coefficient and permeability. Additionally, lattice GCMC and classical density functional theory (cDFT) are also highlighted for computational screening of MOFs in this review. Machine learning consisting of various algorithms is a recently developed strategy with high efficiency and low computational cost, which is a more powerful and promising technique in future. At last, the investigations on the utilization of HTCS in $\mathrm{CH}_{4}$ storage, $\mathrm{H}_{2}$ storage, $\mathrm{CO}_{2}$ capture and gas separation were outlined. By reviewing the recent research progress in HTCS, we pointed out the current challenges and opportunities for the furture development of HTCS for MOFs, which will be the major engine for the commercialization of MOFs in various applications of interests.
\end{abstract}

Keywords metal-organic framework; computational screening; molecular simulation; machine learning; adsorption and separation

\section{1 引言}

金属有 机 骨架 (Metal-Organic Frameworks, $\mathrm{MOFs})^{[1 \sim 4]}$ 是新近发展起来的一种新型纳米多孔晶体材
料，它是由无机结构单元(金属离子、团簇或链)与有机 配体(羧酸盐、磷酸酯或含氮配体等)连接而成的周期性 网状结构. 与传统多孔材料相比, MOFs 具有更大的比

\footnotetext{
*E-mail: songli@hust.edu.cn

$\dagger$ These authors contributed equally to this work.

Received December 12, 2018; published January 8, 2019.

Project supported by the National Natural Science Foundation of China (No. 51606081) and Double first-class research funding of China-EU Institute for Clean and Renewable Energy (No. ICARE-RP-2018-HYDRO-001).

项目受国家自然科学基金(No. 51606081)和中欧清洁与可再生能源学院双一流研究生教学平台培育基金(No. ICARE-RP-2018-HYDRO-001)资助.
} 
表面积、更高的孔隙率、结构组成可调等优势，因而被

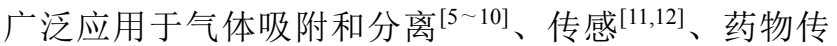
输 ${ }^{[13 \sim 15] \text { 、光采集 }}{ }^{[16 ~ 18]}$ 和催化 ${ }^{[19 \sim 22]}$ 等领域.

随着合成技术的改进和发展，实验制备的 MOFs (experimental MOFs, eMOFs)数量不断增加. 与此同时, 研究人员借助化学理论和计算机技术构建出了大量可 能被合成的 MOFs (hypothetical MOFs, hMOFs). 理论 上, 不同的结构单元、连接方式和拓扑结构的组合可生 成无数的 MOFs 结构. 大量 MOFs 结构的存在也为高效 MOFs 吸附剂的研发带来很多困难. 实验制备和测试一 种 MOF 的性能需要花费一定的时间和经济成本, 如此 庞大的 MOFs 数量意味着漫长的实验周期和高昂的研发 成本; 而实验制备与测试数百万种 MOFs 也是不切实际 的. 近年来, 一些研究者开始尝试理论计算的方法对大 量 MOFs 的吸附性能进行评价和篎选, 并进行实验验证, 从而加速高效 MOFs 的研发进程. 这方面的研究被列入 了美国 “材料基因组计划” (Materials Genome Initiative, MGI), 该计划通过将理论、计算、合成和材料特性结合, 提高新材料研发的效率，旨在实现 “发现、开发和部署 新材料的速度达到目前的两倍” 的目标 ${ }^{[23 ~ 25]}$. 在过去的 数十年中, 高通量计算笁选(High-Throughput Computational Screening, HTCS)已成为最有效的 MOFs 研发技 术. 高通量计算篮选通过对成千上万种 MOFs 材料进行 特定性能的快速评价, 从而篮选出最具应用前景的候选 MOFs, 并且通过数据挖掘技术揭示材料的构效关系 (Quantitative Structure-Property Relationship, QSPR)模 型, 从而用于指导新一代高性能 MOFs 的理性设计与制 备. 迄今, HTCS 技术已被成功用于 $\mathrm{CH}_{4}$ 储存 ${ }^{[26 ~ 28]} 、 \mathrm{H}_{2}$ 的储存 ${ }^{[23,29,30]} 、 \mathrm{CO}_{2}$ 捕捉 ${ }^{[31,32]}$ 和气体分离 ${ }^{[33 ~ 36]}$ 等领域.

MOFs 材料数据库是高通量计算篎选的基础. 目前, 高通量计算篎选主要采用由实验制备的 eMOFs 组成的 eMOFs 数据库和由理论构建的 hMOFs 组成的 hMOFs 数据库. 按照具体篎选方法, 高通量计算篎选又可以划 分为基于分子模拟和基于机器学习算法的高通量计算 笁选两大类. 总结了近年来高通量计算篎选中所使用的 数据库种类、笁选方法及其在吸附分离领域取得的研究 进展. 高通量篎选技术所使用的 MOFs 数据库将在第 2 节中介绍; 第 3 节主要介绍高通量计算篮选中用到的分 子模拟和机器学习方法; 第 4 节总结了近年来高通量计 算篎选技术在 MOFs 的 $\mathrm{CH}_{4}$ 储存、 $\mathrm{H}_{2}$ 储存、 $\mathrm{CO}_{2}$ 捕捉和 气体分离等方面的应用; 最后, 我们将讨论 MOFs 高通 量计算篮选技术的未来发展方向及其所面临的挑战.

\section{MOFs 数据库}

随着 MOFs 高通量计算笁选技术的出现, 由实验合 成或计算机构建的 MOFs 材料数据库成为高通量计算篎 选快速发展的基础. MOFs 数据库基本可划分为两类: (1)由实验合成的 MOFs 组成的 $\mathrm{eMOFs}$ 数据库, 如 Chung
等 ${ }^{[27]}$ 建立的 CoRE MOF (Computation-Ready, Experimental MOFs)数据库; (2)根据化学理论, 由计算机设计 的可能被合成的 hMOFs 数据库, 如 Wilmer 等 ${ }^{[34]}$ 在 2011 年建立的 hMOFs 数据库. 作为高通量计算篎选的基础, MOFs 的各种数据库已被广泛应用于气体的吸附、分离 和储存领域 ${ }^{[37]}$.

\section{1 eMOFs 数据库}

eMOFs 数据库主要从已知晶体结构实验数据库(如 Cambridge Structure Database, CSD)中提取、构建一系列 接近完美的、可以立即用于分子模拟或可视化的 MOFs 结构. 最新研究表明由剑桥晶体数据中心(Cambridge Crystallographic Data Centre, CCDC)维护和更新的结构 数据库中 MOFs 的数量已增至 69666 种, 其中 “有序 MOF 子集” 包含 54808 种有序的 MOFs 结构 ${ }^{[38]}$. 然而, 其中仍有许多 MOFs 的晶体结构包含溶剂分子或存在不 同程度的原子混乱、氢原子缺失和原子重叠等问题，导 致不能直接将其应用到高通量篎选计算中. 2012 年，美 国佐治亚理工大学的 Sholl 课题组 ${ }^{[39]}$ 率先从 $\mathrm{CSD}^{[40,41]}$ 中 提取出约 30000 种 MOFs, 通过计算原子间键长、材料

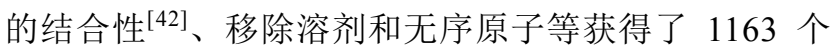
MOFs 结构, 并将其用于 $\mathrm{CO}_{2} / \mathrm{N}_{2}$ 分离的高通量篎选研 究. 2013 年, 美国密歇根大学 Siegel 课题组 ${ }^{[43]}$ 从 CSD 中 收集并整理了 4000 种 $\mathrm{eMOFs}$ 用于储氢 MOFs 的高通量 计算笁选. 2014 年, 美国西北大学 Snurr 课题组 ${ }^{[27]}$ 也基 于 CSD 建立了 CoRE MOF 数据库, 主要包括以下几个 步骤(图 1): (1)基于化学成分确定结构数据库中潜在的 MOF 结构, 选出 60000 种候选材料; (2)消除一维聚合物 和二维氢键网络结构等非 MOFs 材料, 保留三维的 MOFs, 剩余 20000 余种候选材料; (3)移除晶体位点被占 据、结构框架混乱的 MOFs, 确保 MOF 结构呈电中性; (4)清除 MOF 框架中残余的溶剂, 获得 5109 种 MOFs. 随后, Snurr 课题组持续更新 CoRE MOF 数据库, 2016 年

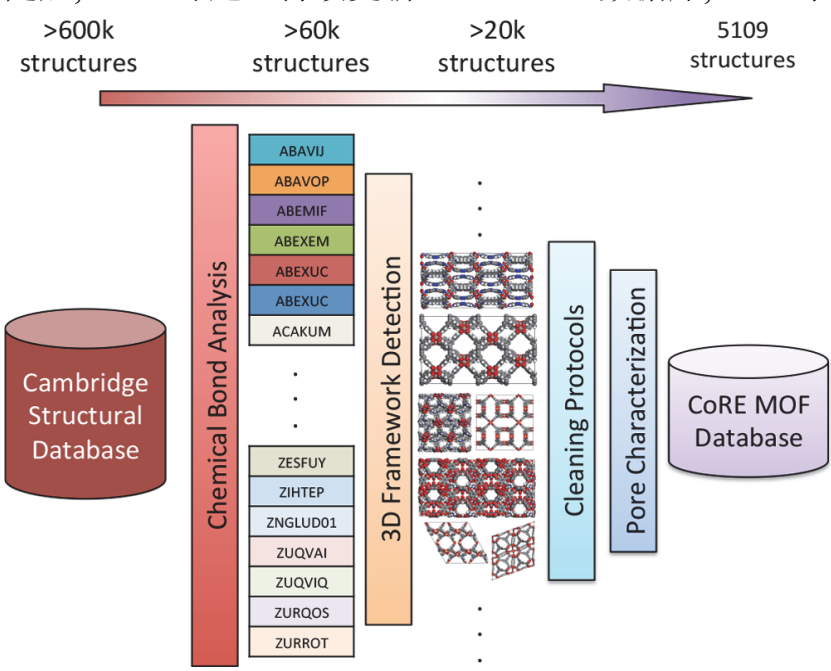

图 1 CoRE MOF 数据库的构建过程示意图 ${ }^{[27]}$

Figure 1 Schematic illustration of CoRE MOF database construction ${ }^{[27]}$ 
表 1 高通量计算篎选中使用的 $\mathrm{eMOFs}$ 数据库

Table 1 Experimental MOF (eMOF) databases for high-throughput computational screening

\begin{tabular}{lclc}
\hline \multicolumn{1}{c}{ Style } & Year & \multicolumn{1}{c}{ Database } & Literature \\
\hline \multirow{4}{*}{ eMOF } & 2012 & 1163 eMOFs from CSD & {$[39]$} \\
Databases & 2013 & 4026 eMOFs from CSD & {$[43]$} \\
& 2012 & 105 eMOFs from literatures & {$[33]$} \\
& 2014 & 151 eMOFs from literatures & {$[44]$} \\
& 2014 & CoRE MOF Database & {$[27]$} \\
& 2016 & CoRE MOF V2.0 Database & {$[45]$} \\
\hline & 2011 & hMOF database from NU & {$[34]$} \\
& 2012 & ZIF database & {$[46]$} \\
hMOF & 2014 & 18383 hMOFs and PAFs & {$[23]$} \\
Databases & 2014 & Zrom Colón & {$[26]$} \\
& 2014 & 324500 hMOFs database & {$[47]$} \\
& 2015 & nandez & {$[46$ hMOFs from McDaniel } \\
& 2016 & 13512 hMOFs from & {$[49]$} \\
& 2017 & Gómez-Gualdrón & {$[32]$} \\
\hline Hybrid eMOF & \multirow{2}{*}{ MMOFOF database } & {$[50]$} \\
and hMOF & 2017 & CoRE MOF database & \\
Databases & & &
\end{tabular}

数据库 $(\mathrm{CoRE} \text { MOF V2.0 })^{[45]}$ 的 $\mathrm{eMOFs}$ 数量上升至 6013 个, 已被用于天然气净化 MOFs 的篮选 ${ }^{[51]}$; 预计 2019 年 CoRE MOF 数据库中的 $\mathrm{eMOFs}$ 数量有望突破 15000 种. 近年来用于高通量计算篮选的 eMOFs 数据库见表 1.

\section{2 hMOFs 数据库}

除了 $\mathrm{eMOF}$ 数据库之外, 高通量计算篮分研究也使 用计算机构建的 hMOFs 数据库. 利用 MOFs 的结构砌 块特性, 采用大量的次级结构单元(Secondary Building Units, SBUs)(金属团簇和有机配体)和不同的连接方式, 能够在计算机上生成各种各样的 MOFs 晶体结构. 已有 研究 ${ }^{[34,41,46,52 \sim 54]}$ 报道了 “自顶至底” (from top to bottom)(图 2)和 “自底至顶” (from bottom to top)(图 3)两种

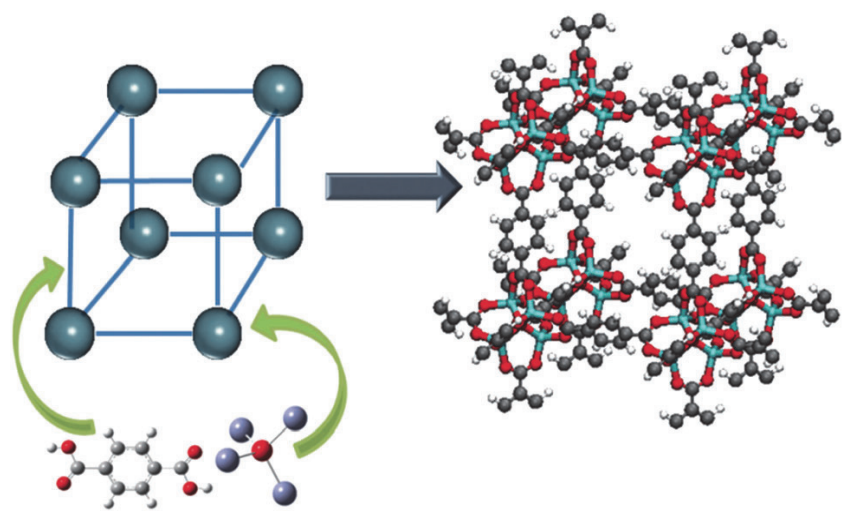

图 2 “自顶至底” 方法示意图. 图中为 $\mathrm{Zn}_{4} \mathrm{O}$ 复合物和对苯二甲酸配 体分别映射到节点和 pcu网络的边缘上, 形成 MOF-5. 灰色为碳原子, 红色为氧原子, 白色为氢原子, 亮蓝色为锌原子 ${ }^{[55]}$

Figure 2 Schematic of the top-down approach, where a $\mathrm{Zn}_{4} \mathrm{O}$ complex and a terephthalic acid linker are respectively mapped onto the node and the edge of a pcu net, forming MOF-5. $\mathrm{C}=$ gray, $\mathrm{O}=$ red, $\mathrm{H}=$ white, $\mathrm{Zn}$ $=$ light blue ${ }^{[55]}$
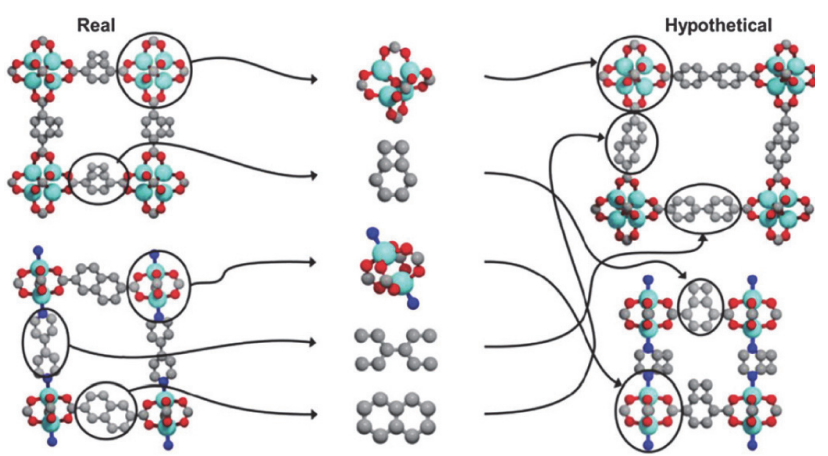

图 3 “自底至顶”方法示意图. 利用 $X$ 射线衍射数据得到已有 MOFs 的晶体结构(左), 然后将其划分为结构砌块(中), 并重新组合成新的 假设 MOFs (右). 灰色、红色、蓝色和绿色的球分别代表碳、氧、氮 和锌原子[34]

Figure 3 Schematic of the bottom-up approach. Crystal structures of existing MOFs were obtained from X-ray diffraction data (left) and subsequently divided into building blocks (middle) that then could be recombined to form new, hypothetical MOFs (right). Gray, red, blue and turquoise spheres represent carbon, oxygen, nitrogen and zinc atoms, respectively $^{[34]}$

构建 hMOFs 的方法. “自顶至底”的方法 ${ }^{[5]}$ 是指将 SBUs 放置在给定的拓扑结构或连接位点上以构建出 MOF 结 构. “自底至顶” ${ }^{[56]}$ 的方法则是将 SBUs 顺序连接直到 形成所有可能的周期性 MOF 结构. 在 hMOF 的生成过 程中，可以使用多种 SBUs，因此, hMOFs 所具有的模块 化特性和连接方式的多样性使得它们理论上存在大量 的可能的结构.

$\mathrm{hMOF}$ 数据库通常具有更明显的规律性, 从而可以 从中获得更全面的构效关系, 并对笁选出的具有优异性 能的 hMOFs 进行实验制备 ${ }^{[57]} .2011$ 年, 美国西北大学 Snurr 课题组 ${ }^{[34]}$ 首先使用 102 个 SBUs, 采用“自底至顶” 的方法, 建立了包含 137953 个 hMOFs 的数据库(hMOFs from the Northwestern University, hMOFs form NU). 其 中每个 $\mathrm{hMOF}$ 仅包含 1 种金属团簇和 $1 \sim 2$ 种有机配体, 以及 1 种官能团. 篮选出的 hMOFs 的 $\mathrm{CH}_{4}$ 等温吸附曲 线与实验测量结果相吻合, 验证了 $\mathrm{hMOFs}$ 数据库用于 高通量计算篮选的可靠性. 2012 年, 美国加州大学伯克 利分校的 Smit 课题组 ${ }^{[46]}$ 基于包含 30 万种计算机构建的 沸石结构数据库 ${ }^{[58,59],}$, 利用锌团簇取代沸石中的硅原子 并通过咪唑酯(imidazolate)配体来连接氧原子, 获得了 ZIF (Zeolitic Imidazolate Framework)数据库. 此外, 研 究人员还通过不同的方法构建了适用于高通量计算篮 分的其他类型的 $\mathrm{hMOF}$ 数据库 ${ }^{[23,26,32,47,49]}$. 如 Gómez-Gualdrón 等 ${ }^{[49]}$ 为研究得到在低温条件下适合储 存 $\mathrm{H}_{2}$ 的材料, 通过 “自顶至底” 的方法将 41 个 SBUs 连接得到 13512 个 hMOFs 结构. 我们课题组 ${ }^{[32]}$ 通过“自 底至顶” 方法构建了由 1 种金属、3 种有机配体和 3 种 官能团组成的多元(multivariate, MTV) MOF, 生成了 10995 种 MTV-MOFs, 并将其用于 $\mathrm{CO}_{2} / \mathrm{N}_{2}$ 的分离及其构 效关系分析. 更多 hMOFs 数据库见表 1 . 
值得指出的是, 除了单纯的 eMOFs 和 hMOFs 数据 库外, 也有研究 ${ }^{[48,50]}$ 对 $\mathrm{eMOFs}$ 和 $\mathrm{hMOFs}$ 数据库同时进 行篎选, 从而增加研究样本的数量. Qiao 等 ${ }^{[50]}$ 利用高通 量计算篎选的方法，同时对 hMOFs 数据库(包含 137953 个 $\mathrm{hMOFs}$ ) 和 CoRE MOF 数据库(包含 4767 个 eMOFs) 进行篎选, 最终获得了能在空气中对硫醇具有良好捕获 性能的 MOFs, 并探究了其构效关系. 其他相关研究见 表 1 .

\subsection{MOFs 数据库存在的问题}

以上内容总结了常用 MOFs 数据库以及近年的研究 进展. 尽管目前研究人员已经在数据库的构建方面取得 了重大的进展, 但仍存在很多问题亟待解决. 首先对于 eMOFs 数据库如 CoRE MOF 数据库, 在使用时出现了 一些问题, 如 “清理” 后的 eMOF 结构与实验制备的 MOF 结构之间存在差异, 可能导致理论与实验结果之 间存在差别. 在生成 $\mathrm{eMOF}$ 数据库的过程中引入了自动 移除溶剂分子或清除无序原子等步骤, 但是这一步骤不 是适用于所有情况. 例如: 假设所有溶剂分子都可以从 结构中移除, 这在某些情况下是正确的; 但存在一些有 溶剂的 MOFs, 在这种情况下框架的稳定性依赖于溶剂 分子而存在(如水、甲醇等), 此时移除溶剂分子将会导 致与实验不符的计算结果 ${ }^{[60 ~ 62]}$. 其次, 对于 hMOFs 数 据库, hMOFs 能否被实验成功制备尚存疑虑. 尽管有部 分 hMOFs 在实验中已经被成功合成, 但是大部分通过 计算机设计的 hMOFs 的实验可行性和设计合理性仍存 在疑问 ${ }^{[63]}$. 已报道的 hMOFs 中能够被实验合成并在实 际应用中具有所需的机械稳定性、热稳定性和化学稳定 性的 hMOF 数量尚不明确, 这些方面都有待进一步研 究.

\section{3 筛选方法}

MOFs 的高通量计算笁选方法主要包括两类: 最为 常用的基于分子模拟的高通量计算篎选方法和近年来 日益受到关注的基于机器学习(Machine Learning, ML) 的篮选方法. 基于分子模拟的高通量计算笁选的一般流 程 ${ }^{[64]}$ 如图 4 所示. 首先, 采用 Zeo $++^{[41]}$ 、Poreblazer ${ }^{[65]}$ 、 MOFomics 或 ZEOMICS ${ }^{[66]}$ 和 TOPOS ${ }^{[67]}$ 等计算数据库中 MOFs 的最大自由直径(Largest Cavity Diameter, LCD)、 受限孔直径(Pore Limiting Diameter, PLD)、有效孔容 (Available Pore Volume)、可接触比表面积(Accessible Surface Area, ASA) 以及拓扑结构等信息, 排除比表面积 过低和孔径过小的 MOFs, 降低计算样本量. 之后, 通 过分子模拟方法对 MOFs 的吸附或扩散性能进行模拟, 结合实际应用条件, 根据 MOFs 性能的各项衡量指标进 行综合评价和篎选, 篎选出的高性能 MOFs 可进一步用 于实验合成与性能测试. 同时, 根据以上获得 MOFs 结 构特征和性能数据, 采用数据分析方法探究 MOFs 的构 效关系模型, 用以指导新一代高性能 MOFs 的理性设计.
近年来, 随着 MOFs 数目的快速增长, 基于分子模拟的 高通量计算笁选难以满足日益增加的需求, 基于机器学 习的高通量计算篮选逐渐受到重视. 首先, 获得数据库 中部分 MOFs 的结构特征和性能数据作为训练集 (training set); 之后采用机器学习算法获得训练集中 MOFs 的构效关系数学模型; 最后, 利用获得的构效关 系模型预测测试集(test set)即数据库中其余 MOFs 的性 能. 基于机器学习的高通量计算篎选具有笁选速度快和 效率高的优势，因而近年来日益受到重视. 本节将对以 上方法进行简要的介绍.
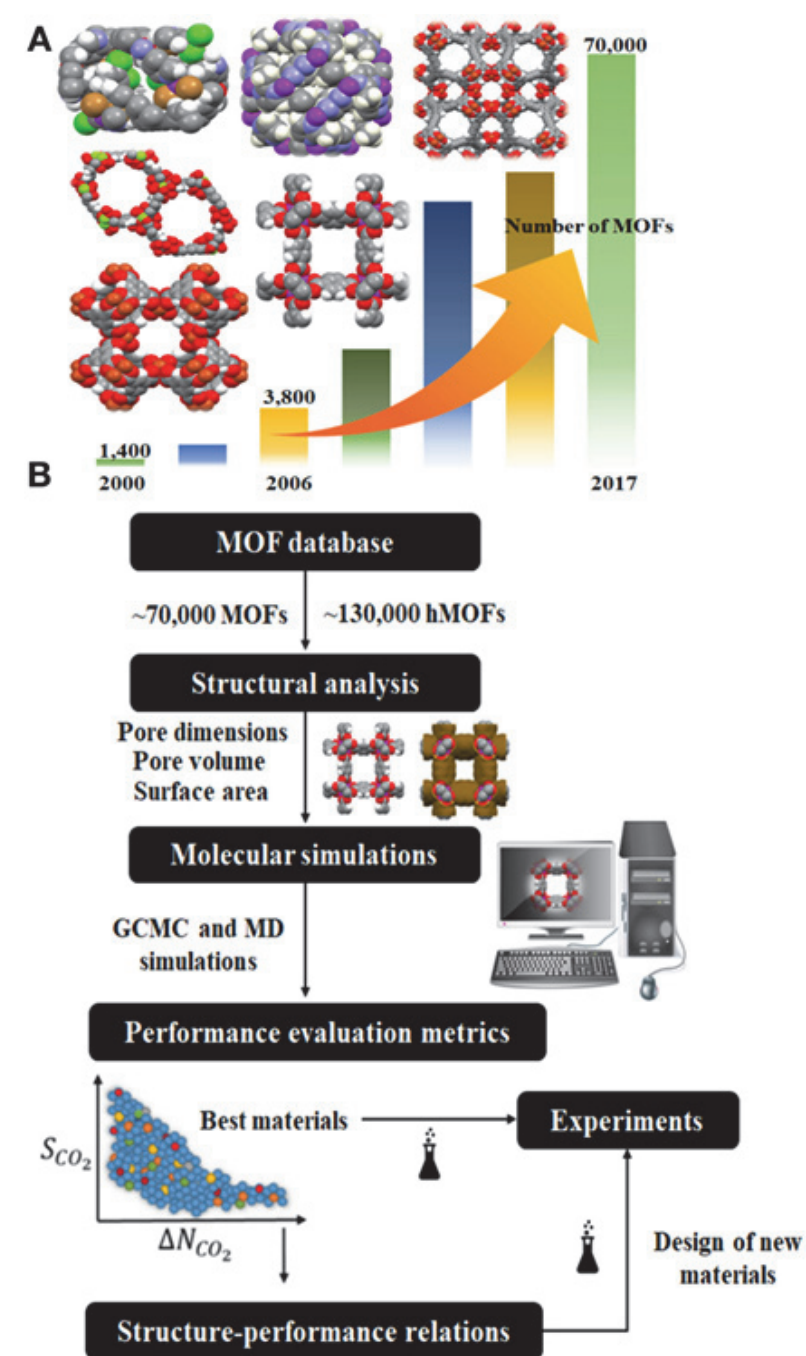

图 4 (A) eMOFs 的数量快速增加, (B)高通量计算篮选方法示意图 ${ }^{[64]}$ Figure 4 Schematic diagram of (A) the rapid increase in the number of synthesized metal organic frameworks, (B) high-throughput computational screening methodology ${ }^{[64]}$

\section{1 分子模拟}

高通量计算篮选中常用的分子模拟方法为巨正则 蒙特卡洛(Grand Canonical Monte Carlo, GCMC)模拟和 分子动力学(Molecular Dynamics, MD)模拟两种. GCMC 分子模拟通过对气体分子在 MOFs 中的插入、删除、移 动和旋转等运动来获得相平衡, 从而获得 MOFs 的吸附 性能，包括吸附量、选择性、吸附热和吸附曲线这类平 
衡态吸附性能. MD 模拟根据牛顿力学原理, 通过相互 作用势解出动力学参数, 而后得到分子运动轨迹, 以此 来获得扩散系数等动力学特征. 对于不同外部环境, 分 子模拟可采用不同的系综来实现.

在分子模拟中, 采用合适的力场描述相互作用是模 拟能准确反映出各项性能的前提条件 ${ }^{[48,68 \sim 70]}$. 近年来, 高通量篮选采用的模拟方法与力场总结在表 2 中. 目前, 对于 MOFs 在变压吸附领域的笁选, 以 GCMC 分子模拟 为主; 而在膜分离领域, 则需要 GCMC 和 MD 分子模拟 相结合来预测分离性能. 当 GCMC 和 MD 相结合时, 一 般需要采用相同的力场. 在 GCMC 和 MD 分子模拟中, 相互作用可以分为 MOF-MOF 之间的相互作用、MOF气体相互作用以及气体-气体相互作用. 在一般的高通 量篎选中, MOFs 采用固定的框架结构, 此时不考虑 MOFs 之间的相互作用. 而气体一气体相互作用仅由气 体分子力场决定, 因此, 如何准确描述 MOFs 与气体分 子之间的相互作用至关重要. MOF-气体相互作用包括 Lennard-Jones 作用势(Lennard-Jones Interaction, LJ)和库 伦作用势(Coulombic Interaction)两部分 ${ }^{[55]}$.

由于 MOF 数据库中结构繁多, 针对具体结构开发 力场难度很高, 因此, 基于 GCMC 和 MD 的高通量笁选 主要采用 Universal (Universal Force Field, UFF) ${ }^{[71]}$ 和 Dreiding (Dreiding Force Field, DFF) ${ }^{[72]}$ 这类通用 LJ 力场. Grajciar 等 ${ }^{[73]}$ 计算了基于 UFF 力场的 HKUST-1 和 $\mathrm{CO}_{2}$ 之间的相互作用, 并与密度泛函理论(Density Functional Theory, DFT)的计算结果相比较, 发现其偏差在可接受 范围内. MOF-气体分子的库伦作用势一般通过点电荷 近似算法获得, MOFs 具体采用的点电荷计算方法一般 是由气体分子的极性决定. 对于库仑作用力极弱的气体
如稀有气体和 $\mathrm{CH}_{4}$, 一般忽略 MOFs 的库伦作用. 而对 于极性较弱的气体分子如 $\mathrm{H}_{2} 、 \mathrm{CO}_{2} 、 \mathrm{~N}_{2}$ 、乙烯和乙炔等, 基于原子电负性计算的电荷平衡(Charge Equilibration,

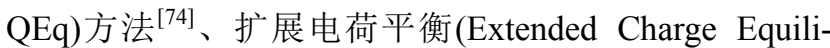
bration, EQeq) 方法 ${ }^{[75]}$ 和基于连接性的原子贡献 (Connectivity-Based Atom Contribution Method, CBAC) 方法 ${ }^{[76]}$ 在笁选中较为常用. 然而, Qeq 方法很可能会高 估卤族元素的电荷，因此导致对 $-\mathrm{F} 、-\mathrm{Cl}$ 等官能团修饰的 MOFs 与气体分子之间的相互作用的计算误差 ${ }^{[77]}$. 尽管 可能存在以上问题，由于 Qeq 方法具有计算速度快、对 计算资源需求极低等优势，因而在高通量计算笁选中得 到广泛的应用. 对于极性较强的气体分子如水 ${ }^{[78,79]}$ 或 醇, MOF 点电荷的准确程度至关重要. 一般采用基于 DFT 的计算方法. 首先采用如 VASP(Vienna Ab initio Software Package $)^{[80 \sim 83]}$ 的 DFT 计算软件获得 MOFs 的电

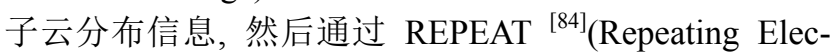
trostatic Potential Extracted Atomic)或 DDEC (Density Derived Electrostatic and Chemical $)^{[85]}$ 方法拟合出点电 荷. Nazarian 等 ${ }^{[86]}$ 比较了 DDEC 和 EQeq 方法计算的 CoRE MOF 数据库的点电荷, 发现 EQeq 方法倾向于高 估碱金属和稀土金属元素的电荷, 而低估碱土金属的电 荷. 另一种常用的方法是采用 Gaussian 中的 ChelpG 方 法分别计算金属团簇与配体的点电荷，再将其分配至 MOFs 晶格单元内. 这种基于非周期性团簇结构的计算 方法忽略了周围环境中其他原子的影响，其准确性有待 商榷. 此外, 研究者还开发了静电势优化(MOFs Electrostatic Potential Optimized, MEPO)的 QEq 方法 ${ }^{[77]}$ 在某 些 MOFs 计算中, 其精度可以与 DFT 计算方法相媲美. 但是对于其他的 MOF, 这种方法的精确度仍尚待商榷.

表 2 MOFs 的高通量计算篮选研究总结

Table 2 Summary of high-throughput computational screening of MOFs for varying applications

\begin{tabular}{|c|c|c|c|c|c|}
\hline Year & Application & Database/number of MOFs & Screening method & $\mathrm{MOF} /$ gas force field & Literature \\
\hline 2012 & $\mathrm{Xe} / \mathrm{Kr}$ separation & hMOF database from NU/137953 & GCMC & $\mathrm{UFF} / \mathrm{Xe}^{[87]}, \mathrm{Kr}^{[88]}$ & [34] \\
\hline 2012 & $\mathrm{CH}_{4} / \mathrm{H}_{2}$ separation & eMOFs from literatures/105 & GCMC & $\begin{array}{l}\text { Hydbrid Dreiding \& UFF/TraPPE } \\
\text { for } \mathrm{CH}_{4} \text { and } \mathrm{H}_{2}{ }^{[89]}\end{array}$ & {$[33]$} \\
\hline 2012 & $\mathrm{CO}_{2}$ capture & hMOF database from NU/137953 & GCMC & UFF/TraPPE & {$[90]$} \\
\hline 2013 & $\mathrm{CO}_{2}$ capture & hMOF database from NU/137953 & GCMC & UFF/in-house FF & {$[31]$} \\
\hline 2013 & $\mathrm{CH}_{4}$ storage & $\begin{array}{l}\text { hMOF database from NU/137953 } \\
\text { (training set: } 10000 \text {, test set: } 127953 \text { ) }\end{array}$ & $\begin{array}{l}\text { ML } \\
(\mathrm{SVM} / \mathrm{MLR} / \mathrm{DT})\end{array}$ & - & [91] \\
\hline 2014 & $\mathrm{H}_{2}$ storage & hMOFs and PAFs from Colón/18383 & GCMC & $\begin{array}{l}\text { UFF/ LJ from Mi- } \\
\text { chels-Degraaff-Tenseldam } \\
\text { model }^{[92]} \text {; charges from } \\
\text { Darkrim-Levesque model }^{[93]}\end{array}$ & {$[23]$} \\
\hline 2014 & $\mathrm{CH}_{4} / \mathrm{CO}_{2}$ separation & eMOFs from literatures/151 & GCMC & $\begin{array}{l}\text { Hydbrid Dreiding \& UFF/TraPPE } \\
\text { for } \mathrm{CH}_{4}, \text { EPM2 for } \mathrm{CO}_{2}\end{array}$ & [44] \\
\hline 2014 & $\mathrm{CH}_{4}$ storage & Zr-hMOFs database/204 & GCMC & UFF/TraPPE & {$[26]$} \\
\hline 2014 & $\mathrm{CH}_{4}$ storage & CoRE MOF Database/5019 & GCMC & UFF/TraPPE & [27] \\
\hline 2014 & $\mathrm{H}_{2}$ storage & hMOF database from NU/137953 & GCMC & $\begin{array}{l}\text { Hybrid Dreiding \& DFT-derived } \\
\text { parameters/Darkrim-Levesque } \\
\text { model }^{[93]}\end{array}$ & [94] \\
\hline 2014 & $\mathrm{CO}_{2}$ capture & $\begin{array}{l}\text { hMOFs from Fernandez/324500 (train- } \\
\text { ing set: } 32450 \text {, test set: } 292050 \text { ) }\end{array}$ & ML (SVM) & 一 & {$[47]$} \\
\hline 2015 & $\mathrm{CH}_{4}$ storage & hMOF database from NU/1200 & $\mathrm{cDFT}$ & UFF/TraPPE & [28] \\
\hline 2015 & $\mathrm{CH}_{4}$ storage & hMOF database from NU/1200 & cDFT vs. GCMC & UFF/TraPPE & {$[95]$} \\
\hline 2015 & $\begin{array}{l}\text { Dibenzothiophene } \\
\text { adsorption }\end{array}$ & hMOF database from NU/1200 & $\mathrm{cDFT}$ & UFF/in-house & [96] \\
\hline
\end{tabular}




\begin{tabular}{|c|c|c|c|c|c|}
\hline Year & Application & Database/number of MOFs & Screening method & $\mathrm{MOF} /$ gas force field & Literature \\
\hline 2015 & $\mathrm{H}_{2}$ storage & hMOF database from NU/1200 & cDFT vs. GCMC & UFF/Buch model ${ }^{[97]}$ & {$[30]$} \\
\hline 2015 & $\begin{array}{l}\mathrm{CO}_{2} \text { capture, } \mathrm{CH}_{4} \\
\text { storage }\end{array}$ & hMOFs from McDaniel/416 & $\begin{array}{l}\text { Lattice GCMC vs. } \\
\text { GCMC }\end{array}$ & $\begin{array}{l}\mathrm{UFF} / \mathrm{EPM} 2 \text { for } \mathrm{CO}_{2} \text {, TraPPE for } \\
\mathrm{CH}_{4}\end{array}$ & [48] \\
\hline 2016 & $\mathrm{CH}_{4} / \mathrm{H}_{2}$ separation & hMOF database from NU/1200 & cDFT & UFF \& Dreiding/in-house & [98] \\
\hline 2016 & $\mathrm{H}_{2}$ storage & hMOF database from NU/137953 & GCMC & $\begin{array}{l}\text { UFF/Darkrim-Levesque model }{ }^{[93]} \\
\text { UFF/LJ from Mi- }\end{array}$ & [29] \\
\hline 2016 & $\mathrm{H}_{2}$ storage & hMOFs from Gómez-Gualdrón/13512 & GCMC & $\begin{array}{l}\text { chels-Degraaff-Tenseldam } \\
\text { model }^{[92]} \text {; charges from } \\
\text { Darkrim-Levesque model }^{[93]}\end{array}$ & [49] \\
\hline 2016 & $\begin{array}{l}\mathrm{CO}_{2} / \mathrm{N}_{2} / \mathrm{H}_{2} \mathrm{O} \text { separa- } \\
\text { tion }\end{array}$ & CoRE MOF Database/5019 & $K_{\mathrm{H}}+\mathrm{GCMC}$ & $\begin{array}{l}\mathrm{UFF} / \mathrm{TraPPE} \text { for } \mathrm{CO}_{2} \text { and } \mathrm{N}_{2}, \\
\text { Tip4p for } \mathrm{H}_{2} \mathrm{O}\end{array}$ & [99] \\
\hline 2016 & $\begin{array}{l}\mathrm{H}_{2} \mathrm{O} / \mathrm{CH}_{4} / \mathrm{NH}_{3} \text { sep- } \\
\text { aration }\end{array}$ & hMOF database from NU/137953 & $K_{\mathrm{H}}+\mathrm{GCMC}$ & $\begin{array}{l}\mathrm{UFF} / \mathrm{TraPPE} \text { for } \mathrm{CH}_{4} \text { and } \mathrm{NH}_{3} \text {, } \\
\text { Tip4p for } \mathrm{H}_{2} \mathrm{O}\end{array}$ & [100] \\
\hline 2016 & $\begin{array}{l}\mathrm{TBM} / \mathrm{CH}_{4} \text { separa- } \\
\text { tion }\end{array}$ & CoRE MOF Database/4519 & GCMC & UFF/TraPPE & [101] \\
\hline 2016 & $\begin{array}{l}\mathrm{CO}_{2} / \mathrm{N}_{2} / \mathrm{CH}_{4} \text { separa- } \\
\text { tion }\end{array}$ & hMOF database from NU/137953 & $\mathrm{GCMC}+\mathrm{MD}$ & $\mathrm{UFF} /$ TraPPE & {$[102]$} \\
\hline 2016 & $\begin{array}{l}\mathrm{NH}_{3} / \mathrm{CH}_{4} / \mathrm{H}_{2} \mathrm{O} \\
\text { sepraration }\end{array}$ & eMOFs from CSD/1163 & $K_{\mathrm{H}}+\mathrm{GCMC}$ & $\begin{array}{l}\mathrm{UFF} / \text { TraPPE for } \mathrm{NH}_{3} / \mathrm{CH}_{4} \text {, Tip } 4 p \\
\text { for } \mathrm{H}_{2} \mathrm{O}\end{array}$ & [39] \\
\hline 2016 & $\begin{array}{l}\mathrm{C}_{2} \mathrm{H}_{6} / \mathrm{C}_{2} \mathrm{H}_{4} \text { separa- } \\
\text { tion, } \mathrm{C}_{2} \mathrm{H}_{6} / \mathrm{CH}_{4} \\
\text { separation }\end{array}$ & $\begin{array}{l}\text { CoRE MOF database } / 233+\text { eMOFs from } \\
\text { literatures } / 45\end{array}$ & $\mathrm{GCMC}+\mathrm{MD}$ & Hybrid Dreiding \& UFF/TraPPE & {$[103]$} \\
\hline 2016 & $\mathrm{CH}_{4} / \mathrm{H}_{2}$ separation & CoRE MOF database $/ 250$ & $\mathrm{GCMC}+\mathrm{MD}$ & Hybrid Dreiding \& UFF/TraPPE & {$[104]$} \\
\hline 2016 & $\begin{array}{l}\mathrm{CH}_{4} / \mathrm{CO}_{2} \text { purifica- } \\
\text { tion }\end{array}$ & $\begin{array}{l}\text { hMOFs from Fernandez/324500 (train- } \\
\text { ing set: } 32450 \text {, test set: } 292050 \text { ) }\end{array}$ & ML (DT/SVM) & - & [105] \\
\hline 2016 & $\mathrm{CO}_{2} / \mathrm{N}_{2}$ separation & hMOF database from NU/137953 & $\begin{array}{l}\text { ML } \\
\text { (MLR/DT/kNN/SV } \\
\text { M/ANN/RF) }\end{array}$ & - & [106] \\
\hline 2016 & $\mathrm{CO}_{2}$ capture & hMOF database from NU/55163 & ML (GA) & - & [107] \\
\hline 2017 & $\mathrm{CO}_{2}$ capture & MTV-MOF database/10995 & GCMC & UFF/TraPPE & {$[32]$} \\
\hline 2017 & $\begin{array}{l}\text { Hexane/heptane } \\
\text { separation }\end{array}$ & $\begin{array}{l}\text { CoRE MOF database } / 5109+\text { IZA data- } \\
\text { base } / 402\end{array}$ & GCMC & UFF/TraPPE-UA & [108] \\
\hline 2017 & $\begin{array}{l}\mathrm{Xe} / \mathrm{Kr}, \mathrm{Xe} / \mathrm{Ar} \\
\mathrm{Xe} / \mathrm{Rn} \text { separation }\end{array}$ & CoRE MOF database/117 & $\mathrm{GCMC}+\mathrm{MD}$ & $\mathrm{UFF} / \mathrm{Xe}, \mathrm{Kr}, \mathrm{Ar}, \mathrm{Rn}^{[109,110]}$ & {$[35]$} \\
\hline 2017 & Thiol capture & $\begin{array}{l}\text { hMOF database from NU/137953+ } \\
\text { CoRE MOF database/4764 }\end{array}$ & GCMC & $\mathrm{UFF} /$ TraPPE & {$[50]$} \\
\hline 2017 & $\mathrm{CH}_{4}$ storage & $\begin{array}{l}\text { hMOF database from NU/130398 } \\
\text { (training set: } 10433 \text {, test set: } 119965 \text { ) }\end{array}$ & ML (DT/SVM/RF) & - & [111] \\
\hline 2017 & $\begin{array}{l}\mathrm{H}_{2} \text { storage, } \mathrm{CO}_{2} \\
\text { capture }\end{array}$ & eMOFs from literatures $/ 100$ & $\begin{array}{l}\text { ML (Just Add Data } \\
\text { V0.6 tool) }\end{array}$ & - & [112] \\
\hline 2018 & $\mathrm{He} / \mathrm{CH}_{4}$ separation & CoRE MOF database/139 & $\mathrm{GCMC}+\mathrm{MD}$ & Hybrid Dreiding \& UFF/TraPPE & [113] \\
\hline 2018 & $\mathrm{CH}_{4} / \mathrm{H}_{2}$ separation & eMOFs from CSD/69666 & GCMC & $\mathrm{UFF} / \mathrm{CH}_{4}{ }^{[114]}, \mathrm{H}_{2}{ }^{[115]}$ & [116] \\
\hline 2018 & $\mathrm{CO}_{2} / \mathrm{N}_{2}$ separation & $\begin{array}{l}\text { hMOF database from NU/110871+ } \\
\text { CoRE MOF database/2017 }\end{array}$ & $K_{\mathrm{H}}+\mathrm{MD}$ & $\mathrm{UFF} /$ TraPPE & [117] \\
\hline 2018 & $\begin{array}{l}\mathrm{CO}_{2} / \mathrm{N}_{2} / \mathrm{CH}_{4} \text { separa- } \\
\text { tion }\end{array}$ & CoRE MOF database/4764 & $\mathrm{GCMC}+\mathrm{MD}$ & $\mathrm{UFF} /$ TraPPE & {$[118]$} \\
\hline 2018 & $\mathrm{CO}_{2}$ capture & $\mathrm{hMOFs} / 400$ & $\begin{array}{l}\mathrm{DFT}+\mathrm{GCMC}+\mathrm{ML} \\
(\mathrm{MLR} / \mathrm{SVM} / \mathrm{DT} / \mathrm{RF})\end{array}$ & UFF/TraPPE & [119] \\
\hline 2018 & $\begin{array}{l}\mathrm{H}_{2} \mathrm{~S} / \mathrm{CO}_{2} / \mathrm{CH}_{4} \text { sepa- } \\
\text { ration }\end{array}$ & CoRE MOF V2.0 Database/6013 & $K_{\mathrm{H}}+\mathrm{GCMC}$ & UFF/TraPPE & [51] \\
\hline
\end{tabular}

对于气体分子的力场, $\mathrm{CH}_{4} 、 \mathrm{CO}_{2} 、 \mathrm{~N}_{2} 、 \mathrm{O}_{2}$ 及各类烷 烃通常采用 TraPPE (Transferable Potentials for Phase Equilibria)力场 ${ }^{[120]}$, 包括全原子的 TraPPE-EH 和粗粒化 (Coarse-grained)的 TraPPE-UA. 也有学者在 $\mathrm{CO}_{2}$ 模拟中 采用 EPM2 (Elementary Physical Model) 力场 ${ }^{[121]}$, 因为 它可以较好地描述 $\mathrm{CO}_{2}$ 相变中物性参数的变化. 水分子 模拟中, Tip4p $\mathrm{p}^{[122]}$ 和 Tip4p-Ew ${ }^{[123]}$ 模型最为常用. $\mathrm{H}_{2}$ 力场 种类更多, 主要包括 TraPPE、 Michels-DegraaffTenseldam model ${ }^{[92]} 、$ Darkrim-Levesque model ${ }^{[93]}$ 和 Buch model ${ }^{[97]}$ 等, 一般会根据需要进行选取或组合.
除了以上提到的 GCMC 和 MD 方法, 为加快计算速 度, 基于经典密度泛函的 cDFT (classical DFT)和基于 GCMC 的 lattice GCMC 在 MOFs 的高通量篮选中也有 应用. 两种方法都将 MOF 吸附空间划分为很多小格子, 仅允许气体分子在格点上移动. 通过事先计算获得的 MOF 在每一格点的作用势快速预测气体分子的吸附曲 线. 同时, cDFT 和 lattice GCMC 都采用粗粒化的气体分 子模型. 基于 $\mathrm{cDFT}$ 和 lattice GCMC 的高通量篎选方法 已成功用于二苯并噻吩的净化 ${ }^{[96]}$ 以及 $\mathrm{CO}_{2}$ 和 $\mathrm{CH}_{4}$ 吸 附 ${ }^{[48]}$. 值得一提的是, 对于水等极性较强的气体分子, 
采用分子模拟需要漫长的计算时间才能达到吸附平衡, 因此通常不直接采用 GCMC 模拟. 而是首先通过 Widom insertion 算法获得不同种类 MOFs 对水分子的亨 利系数(Henry's constant, $K_{\mathrm{H}}$ ), 快速排除对水亲和力过强 或过弱的 MOFs 结构, 再结合 GCMC 模拟对笁选出的少 量 MOFs 进行吸附性能预测.

\section{2 机器学习}

随着 MOFs 数目的不断增长, 基于 $\mathrm{GCMC}$ 的高通量 笁选往往受限于庞大的 MOFs 数据库和有限的计算资 源, 基于机器学习(ML)算法的高通量计算笁选则可以 解决以上问题. 机器学习方法仅采用少量 MOFs 结构特 征及其性能数据作为 “训练集”, 通过各种算法获得 “知 识库”, 即可以有效描述 MOFs 结构特征与其性能之间 的数学模型, 从而进一步用来预测 “测试集” 中的大量 MOFs 的未知性能. 这种方法可以有效减少计算时间, 提高篎选的效率. 目前常用的机器学习方法包括多元线 性回归(Multilinear Regression, MLR)、决策树(Decision Tree, DT)、K 邻近算法(K-Nearest Neighbor, KNN)、支 持向量机(Support Vector Machines, SVMs)、人工神经网 络(Artificial Neural Networks, ANN)、随机森林(Random Forest, RF)和基因遗传算法(Genetic Algorithm, GA)等.

多元线性回归算法(MLR) ${ }^{[91]}$ 是通过线性回归方程 拟合获得两个及两个以上变量与响应变量之间数学关 系的一种算法. 这里的变量主要指 MOFs 的结构特征如 比表面积、孔径和孔体积等, 响应变量主要是指吸附量、

(a)

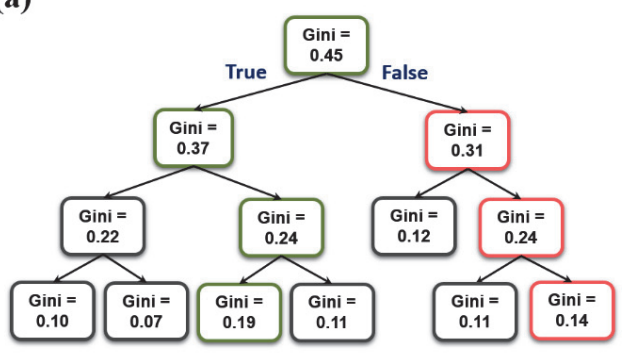

(c)

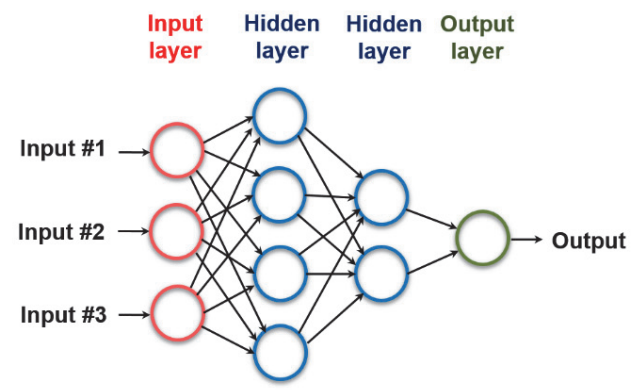

选择性等性能数据. 首先通过对已知 “训练集” 中的数 据进行多元线性拟合获得可以准确描述 MOFs 结构特征 与性能之间的数学模型, 之后输入 “测试集” 中的结构 特征预测其未知性能. 与 MLR 不同, 决策树(DT) ${ }^{[124]}$ 算 法(如图 5 (a)所示)是一种机器学习分类算法, 根据 “测 试集” 中数据的属性, 采用树枝状结构建立决策模型, 可以直观地给出 MOFs 结构特征与性能之间的关系, 便 于理解，然而决策树算法不适合处理大规模数据和连续 变量. 随机森林(Random Forest, RF)算法是一种较为常 用的监督式学习算法, 通过 “训练集” 构建多个决策树 并将其组合到一起，从而获得更准确和稳定的预测结 果. 与 DT 算法类似, $\mathrm{K}$ 邻近算法 $(\mathrm{KNN})$ 也是一种分类算 法, 是通过测量 “训练集” 中不同特征值之间的距离进 行分类的算法，避免了匹配的问题，更为简单，同时 $\mathrm{KNN}$ 通过占优的分类进行决策. 支持向量机 $(\mathrm{SVMs})^{[91]}$ 是一种数据分类导向方法, 原理如图 5 (b) 所示, 它可以 通过数学变换方法将数据集中的结构按一定规律区分 开，适合复杂线性或非线性构效关系，比 MLR、DT 和 KNN 算法更具灵活性, 往往能够获得更准确的预测结 果. 人工神经网络 $(\mathrm{ANN})^{[125]}$ 算法是为了找出各项结构 特征与性能之间的联系, 构建如图 5 (c)中所示的神经网 络, 通过不断调节隐含层中的函数参数, 得到一个适合 的模型来准确预测 “测试集” 的未知性能. 不同于以上 算法, 图 5 (d)所示的基因遗传算法 $(\mathrm{GA})^{[107]}$ 是一种随机 全局搜索并寻找最优解的算法, 类似自然界中基因遗传

(b)

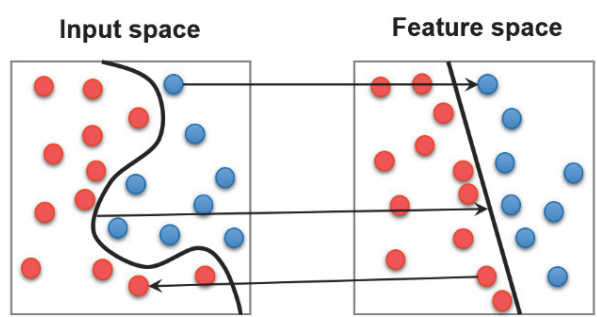

(d)

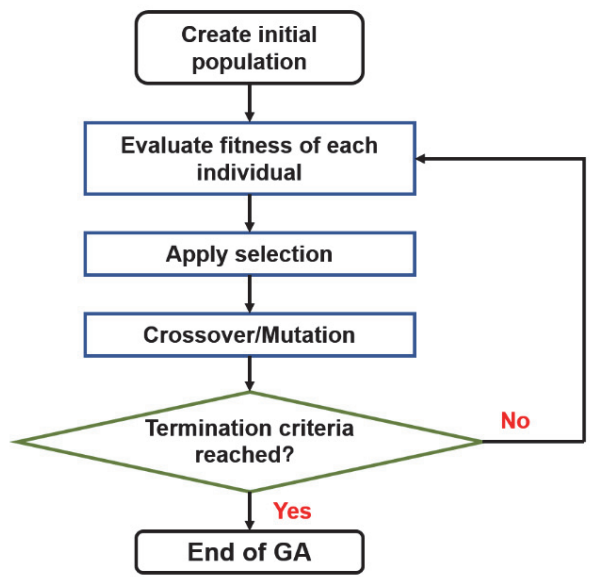

图 5 (a)决策树(DT), (b)支持向量机(SVMS), (c)人工神经网络(ANN)以及(d)基因遗传算法(GA)示意图

Figure 5 Schematic diagram of (a) DT, (b) SVMs, (c) ANN and (d) GA 
的规律, 新结构由上一代高性能的材料的基因遗传而 来, 在多代以后, 能够预测出具有较高性能的 MOFs 结 构. 综上, 基于机器学习的高通量计算篮选通常采用分 子模拟获取的 “训练集” 数据, 以机器学习算法加以分 析和模型构建, 再以获得的构效关系模型准确预测 “测 试集” 中的 MOFs 的性能.

\section{4 高通量節选的应用}

迄今为止, 高通量计算篎选技术已经在 MOFs 的 $\mathrm{CH}_{4}$ 储存、 $\mathrm{H}_{2}$ 储存、 $\mathrm{CO}_{2}$ 捕捉和气体分离等领域均有应 用. 已报道的高通量计算篮选研究多采用 GCMC 分子 模拟方法, 从 MOFs 数据库中笁选出在不同应用中性能 优异的 MOF 结构; 同时, 采用数据挖掘方法对获得的 数据进行构效关系分析, 进一步指导 MOFs 的设计. 此 外, 近期采用机器学习算法实现对 MOFs 的笁选与构效 关系挖掘的研究也有少量报道. 本节主要围绕基于分子 模拟和机器学习算法的高通计算笁选在 MOFs 的 $\mathrm{CH}_{4}$ 储存、 $\mathrm{H}_{2}$ 储存、 $\mathrm{CO}_{2}$ 捕捉和其他气体分离领域的应用进 行介绍.

\section{$4.1 \mathrm{CH}_{4}$ 储存}

近年来, 以 $\mathrm{CH}_{4}$ 为主要成分的天然气被认为是可以 替代汽油的一种极具前景的车用低碳燃料 ${ }^{[126 ~ 128]}$. 但在 常温常压条件下, $\mathrm{CH}_{4}$ 的体积能量密度仅为汽油的 $1 \%$, 天然气储存问题是天然气动力汽车得以广泛应用的关 键 ${ }^{[40]}$. MOFs 由于其超高的比表面积而被认为是储存能 源气体最有前景的材料之一. 高通量计算笁选可以快速 从 MOFs 数据库发现在特定条件下具有优异 $\mathrm{CH}_{4}$ 储存性 能的 MOFs, 获得其构效关系, 并指导高性能 MOFs 的 设计.

2011 年, Wilmer 等 ${ }^{[34}$ 采用 “自底至顶” 的 MOF 生 成方法(图 3)构建了 137953 个 hMOFs, 并在 $298 \mathrm{~K} / 35$ bar 的条件下 (美国能源部规定的 $\mathrm{CH}_{4}$ 储存条件)采用 $\mathrm{GCMC}$ 进行 $\mathrm{CH}_{4}$ 的吸附模拟. 篮选结果表明超过 300 个 hMOFs 的体积吸附量高于当时的 $\mathrm{CH}_{4}$ 储存量最高的 MOF PCN-14 (230 $\left.\mathrm{vol}_{\mathrm{stp}} / \mathrm{vol}\right)$, 远超美国能源部的要求 (180 $\left.\mathrm{vol}_{\mathrm{stp}} / \mathrm{vol}\right)$. 同时, 获得的关于 MOFs 和 $\mathrm{CH}_{4}$ 储存性 能的构效关系表明 $\mathrm{CH}_{4}$ 储存性能优异的 MOFs 的空隙率 约为 0.8 、比表面积为 $2500 \sim 3000 \mathrm{~m}^{2} / \mathrm{g}$ 且孔径为 $4 \sim 8 \AA$ 之间(容纳 $1 \sim 2$ 个 $\mathrm{CH}_{4}$ 分子). 研究还发现吸附量高于 $205 \mathrm{vol}_{\mathrm{stp}} / \mathrm{vol}$ 的 MOFs 中 $75 \%$ 以上含有甲基、乙基或丙 基官能团，并且根据构效关系所设计的 NOTT-107 的 $\mathrm{CH}_{4}$ 储存量高达 $267 \mathrm{vol}_{\mathrm{stp}} / \mathrm{vol}$. 这也是关于 MOFs 的高 通量计算篎选研究的首次报道. 2014 年, 研究人员对 204 个 $\mathrm{Zr}$ 基 hMOFs 进行高通量计算篎选 ${ }^{[26]}$, 寻找一种 稳定且具有高 $\mathrm{CH}_{4}$ 输运能力 (Deliverable Capacity)的 MOF 材料. 耖选出的 NU-800 在 298 K、65-5.8 bar 之间 具有 $167 \mathrm{vol}_{\mathrm{stp}} / \mathrm{vol}$ 的可输运 $\mathrm{CH}_{4}$ 容量, 高于其他 $\mathrm{Zr}$ 基 MOFs.
在对 CoRE MOF 数据库的 $\mathrm{CH}_{4}$ 储存容量的研究中, Chung 等 ${ }^{[27]}$ 对 5109 种 CoRE MOFs 在 298 K/65-5.8 bar(吸附压力: 65 bar; 脱附压力: 5.8 bar)条件下进行了 $\mathrm{CH}_{4}$ 吸附的 GCMC 模拟. 篮选结果表明 MIL-53(Al)具有 最佳的 $\mathrm{CH}_{4}$ 储存能力 (65 bar 时, 储量高达 $267 \mathrm{vol}_{\mathrm{stp}} / \mathrm{vol}$ ). 其构效关系与 hMOFS 数据库的篩选结果基本一致, 如 图 6 所示. 另外, 研究还发现 CoRE MOF 数据库和 hMOFs 数据库的高通量笁选结果都表明具有高效 $\mathrm{CH}_{4}$ 储量的 MOFs 的最佳吸附热为 $10 \sim 15 \mathrm{~kJ} / \mathrm{mol}$.
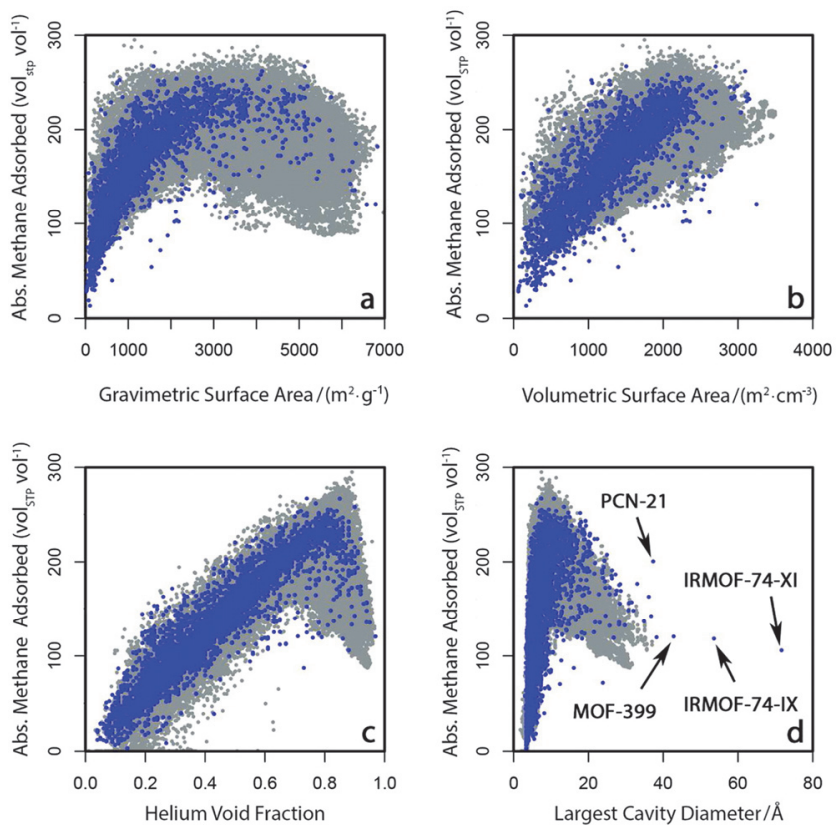

图 $6298 \mathrm{~K} / 65$ bar 条件下 CoRE MOF 数据库(蓝色)和 $\mathrm{hMOF}$ 数据库 (灰色)的绝对 $\mathrm{CH}_{4}$ 吸附量与(a)质量比表面积, (b)体积比表面积, (c)氦 空隙率, (d)优势孔直径的关系图 ${ }^{[27]}$

Figure 6 Absolute methane storage capacity at $298 \mathrm{~K}$ and 65 bar for the CoRE MOF structures (blue) and the hMOF structures (gray) plotted as a function of (a) gravimetric accessible surface area, (b) volumetric accessible surface area, (c) helium void fraction, and (d) largest cavity diameter $^{[27]}$

近年来，基于 $\mathrm{cDFT}$ 的高通量计算篮选也被用于 MOFs 的 $\mathrm{CH}_{4}$ 储存性能的研究. 2015 年, $\mathrm{Fu}$ 等 ${ }^{[28]}$ 对 1200 个 hMOFs 在 $298 \mathrm{~K} / 35-5$ bar(吸附压力: 35 bar; 脱附压 力: 5 bar)条件下分别采用基于四种不同势函数的 $\mathrm{cDFT}$ 和 GCMC 方法计算了 $\mathrm{CH}_{4}$ 的储运能力, 并对比了四种 势函数和 GCMC 的计算结果. 研究结果表明: 基于平均 场近似(Mean-field Approximation, MFA) 函数的 $\mathrm{cDFT}$ 与 $\mathrm{GCMC}$ 的 $\mathrm{CH}_{4}$ 吸附结果最接近(相关系数 $R^{2}=0.99$ ), 是 最佳的可用于 $\mathrm{CH}_{4}$ 储存的纳米多孔材料高通量篮选的 势函数模型. 基于 MFA 的 $\mathrm{cDFT}$ 完成单个甲烷吸附模拟 的平均计算时间为 $2 \mathrm{~min}$ 左右，比传统的 GCMC 模拟快 一个数量级, 因此更加适合包含庞大数目的 MOFs 数据 库的高通量篎选. 此外, 结合过剩熵定标法(Excessentropy Scaling Method), cDFT 还可用于预测多孔材料 中的气体自扩散系数, 这对于实际应用具有重要意义. 
另外, 具有强大数据分析和挖掘能力的机器学习 (ML)方法也已被用来进行高效的 MOFs 高通量篮选研 究. 2013 年, Fernandez 等 ${ }^{[91]}$ 运用不同机器学习算法包括 多元线性回归(MLR)、决策树(DT)和支持向量机(SVMs) 对 hMOFs 数据库的 137953 种 MOFs 进行了有关 $\mathrm{CH}_{4}$ 储存性能的定量构效关系 (Quantitative StructureProperty Relationship, QSPR)分析. 在 298 K/1 bar 和 298 $\mathrm{K} / 35 \mathrm{bar}$ 和 $298 \mathrm{~K} / 100 \mathrm{bar}$ 条件下, 分别使用包含 10000 个 MOFs 的 “训练集” 进行构效关系学习, 并采用获得 的数学模型对包含 127953 个 MOFs 的 “测试集” 进行 预测, 并与 GCMC 的模拟结果对比来评估预测的准确 性. 结果表明, SVMs 算法在预测 $\mathrm{CH}_{4}$ 储存性能方面优 于 DT 和 MLR. SVMs 在 $35 \mathrm{bar}$ 和 $100 \mathrm{bar}$ 条件下的预测 准确率分别为 $85 \%$ 和 $93 \%$. 研究还发现 MOFs 空隙率和 主要孔径是与 $\mathrm{CH}_{4}$ 储存性能最密切相关的性质, 如图 7 所示, 响应面中具有优异 $\mathrm{CH}_{4}$ 储存性能的 $\mathrm{MOFs}$ (图中蓝 色箭头指出)所对应的孔径范围为 $16 \sim 20 \AA$. 值得关注 的是, 预测结果表明可能存在 $\mathrm{CH}_{4}$ 储存性能更好却未包 含在数据库中的 MOFs (图中红色箭头表示). 2017 年, Pardakhti 等 ${ }^{[111]}$ 在 $298 \mathrm{~K} / 35$ bar 条件下采用 DT、泊松回 归(Poisson Regression, PR)、SVMs 和 RF 四种机器学习 方法对包含 130398 个 $\mathrm{hMOFs}$ 的数据库进行了高通量篮 选研究. 研究使用该数据库的 $8 \%$ (10433 个 MOFs) 作为 “训练集”, 剩余的 $92 \%$ (119965 个 MOFs) 作为“测试集”. 与 Fernandez 等 ${ }^{[91]}$ 研究不同, 他们引入了三个变量参数: 结构变量、化学变量和结构/化学混合变量来评估不同机 器学习算法的预测能力. 结果表明, 结构/化学混合变量 组合的方式提高了四种 ML 算法的预测准确性, 而四种 模型中, RF 具有最佳的预测能力. 采用交叉验证方法, $\mathrm{RF}$ 算法对 $\mathrm{CH}_{4}$ 单位质量吸附量预测准确性系数 $R^{2}$ 为 0.98, 平均绝对误差百分比(Mean Absolute Percentage Error, MAPE)仅为 $7.18 \%$; RF 算法对 $\mathrm{CH}_{4}$ 体积吸附量预 测准确性系数 $R^{2}$ 和 MAPE 分别为 0.94 和 $7.54 \%$, 具有 相当高的预测精确度. 更重要的是, 基于 RF 算法对 hMOFs 数据库进行训练和测试的过程仅花费了约 $2 \mathrm{~h}$, 比基于分子模拟的高通量计算篎选提高了几个数量级.

目前, 对于 $\mathrm{MOFs}$ 的 $\mathrm{CH}_{4}$ 储存性能研究主要集中在 高纯度 $\mathrm{CH}_{4}$ 的储存. 而实际上, 商业天然气中还含有少 量高级烃(如乙烷和丙烷), 在多次吸附/脱附循环后, 这 些高级烃可能在 MOFs 中积聚, 导致实际可利用 $\mathrm{CH}_{4}$ 容 量降低. 为了探究少量乙烷和丙烷的存在对 MOFs 的天 然气(Adsorbed Nature Gas, ANG) 储罐可输送 $\mathrm{CH}_{4}$ 容量 的影响, Zhang 等 ${ }^{[95]}$ 开发了一种基于 GCMC 分子模拟和 热力学模型(ANG 模型)的高通量篎选方法, 可以描述 ANG 储罐在充放 $\mathrm{CH}_{4}$ 过程中的杂质气体对其储量的影 响. 该研究以 GCMC 获得的纯组分的 $\mathrm{CH}_{4}$ 、乙烷和丙烷 等温吸附曲线和理想吸附溶液理论(Ideal Adsorption Solution Theory, IAST)计算的混合气体吸附曲线为输入 参数, 通过 ANG 模型在 $298 \mathrm{~K} / 65-1$ bar (吸附 65 bar, 脱
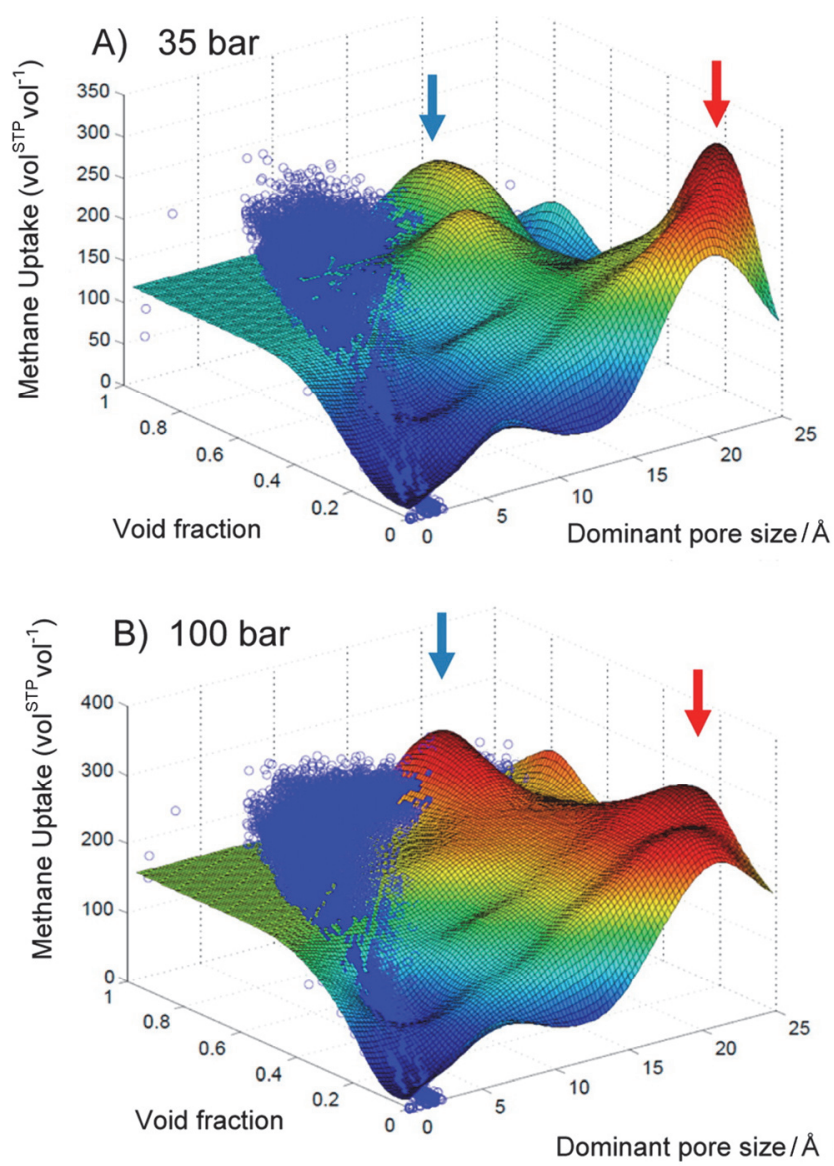

图 7 (A) $298 \mathrm{~K} / 35 \mathrm{bar}$ 和(B) $298 \mathrm{~K} / 100 \mathrm{bar}$ 条件下 SVMs 模型预测的 $\mathrm{CH}_{4}$ 吸附量与空隙率和主要孔径的二维响应曲面. 蓝色点表示 GCMC 模拟吸附量的值; 曲面颜色表示 $\mathrm{CH}_{4}$ 吸附量的值(蓝色表示最低, 红 色表示最高); 蓝色和红色箭头指示响应面上的最大值 ${ }^{[1]}$

Figure 7 Two-dimensional response surfaces of the SVM models for methane storage at $298 \mathrm{~K}$ and (A) 35 bar and (B) 100 bar using void fraction and dominant pore size. The blue dots represent the GCMC simulated uptake values. The color of the surface represents the methane storage value, with blue representing the lowest value and red representing the highest value. Blue and red arrows indicate maxima on the response surface ${ }^{[91]}$

附 1 bar)条件下对 CoRE MOF 数据库中 120 个 MOFs 进 行数学模拟, 超过 200 个吸脱附循环后发现所有 MOFs 的 ANG 储罐的性能在早期循环保持单调下降, 并逐步 达到“循环稳态”. 当气体成分为甲烷 $(96 \%)$ 、乙烷 $(3.3 \%)$ 和丙烷 $(0.7 \%)$ 时, 循环初期具有优异 $\mathrm{CH}_{4}$ 吸附性能的 MOFs 在多个吸脱附循环后性能有所下降. 通过篮选发 现在稳态具有最高 $\mathrm{CH}_{4}$ 储存性能的 MOFs 为 MOF-143, 基于 MOF-143 的稳态 $\mathrm{ANG}$ 储罐的可利用 $\mathrm{CH}_{4}$ 能量密度 可达 $5.43 \mathrm{MJ} / \mathrm{L}$. 因此, 结合 GCMC 分子模拟和 $\mathrm{ANG}$ 数 学模型的高通量笁选方法不失为一种预测 $\mathrm{MOFs}$ 在 $\mathrm{CH}_{4}$ 储罐中的真实吸脱附性能的有效策略.

\section{$4.2 \mathrm{H}_{2}$ 储存}

与 $\mathrm{CH}_{4}$ 相比, $\mathrm{H}_{2}$ 具有更高的单位质量燃烧焓和更洁 净的燃烧产物. 近年来发展的吸附储氢技术具有体积能 量密度大、储存效率高和安全可靠等优点, 其中 MOFs 
材料因其比表面积大、空隙率高、可以快速吸放 $\mathrm{H}_{2}$ 等 优良性质而成为极具前景的储氢材料 ${ }^{[129]} .2015$ 年, 美国 能源部(U.S. Department of Energy, DOE)提出在温度为 $233 \sim 358 \mathrm{~K}$ 区间和压力为 $1 \sim 100$ bar 区间情况下，气态 $\mathrm{H}_{2}$ 的单位质量储量目标为 $5.5 \mathrm{wt} \%$, 单位体积储量目标 $50 \mathrm{~g} / \mathrm{L}^{[130]}$.

迄今, 高通量计算篮选在开发储氢性能优异的 MOFs 材料的研究已有大量报道. 2014 年, Gomez 等 ${ }^{[94]}$ 在 $77 \mathrm{~K}$ 条件下对 137953 个 hMOFs 和其中 1411 个具有 浆轮(Paddle Wheel, PW) 结构的 MOFs (PW-MOFs)进行 了 GCMC 模拟, 以笁选适合用于储氢的 MOFs. 研究发 现有 4 种 MOFs 的 $\mathrm{H}_{2}$ 质量储存量和体积储存量分别达 到 $2.4 \%$ 和 $17.3 \mathrm{~g} / \mathrm{L}$ (77 K/1 bar 条件下), 超过了已报道的 同等条件下储氢量最高的 IRMOF-62 (质量储存量为 1.7\%). 研究还发现 PW-MOFs 中, HKUST-1 (及其同结 构 $\mathrm{Zn}$ 的衍生物) 具有最优的 $\mathrm{H}_{2}$ 吸附性能, 在 $77 \mathrm{~K} / 1 \mathrm{bar}$ 和 $77 \mathrm{~K} / 10$ bar 条件下 $\mathrm{H}_{2}$ 储存量分别可以达到 $1.8 \%$ 和 $4 \%$. Bobbitt 等 ${ }^{[29]}$ 采用 $\mathrm{GCMC}$ 模拟对 $\mathrm{hMOFs}$ 数据库中所 有的 MOFs 在 $77 \mathrm{~K} / 100-2$ bar (吸附 100 bar, 脱附 2 bar) 条件下的 $\mathrm{H}_{2}$ 储运能力进行预测. 研究发现超过 26000 个 MOFs 的 $\mathrm{H}_{2}$ 输运能力超过 $7.5 \mathrm{wt} \%$, 最高为 $24.6 \mathrm{wt} \%$. 有 49 个 MOFs 的体积输运能力超过 $48 \mathrm{~g} / \mathrm{L}$, 其中最大为 $50 \mathrm{~g} / \mathrm{L}$ (质量输运能力为 $12 \mathrm{wt} \%, 100 \mathrm{bar}$ 下绝对吸附量 为 $56.9 \mathrm{~g} / \mathrm{L}$ ), 笁选结果如图 8 所示. 根据所获得构效关 系发现具有优异 $\mathrm{H}_{2}$ 输运能力 MOFs 的最佳空隙率约为 0.9, 孔径为 $12 \sim 15 \AA$, 体积比表面积约为 $2000 \mathrm{~m}^{2} / \mathrm{cm}^{3}$, 最佳吸附热为 $2 \sim 4 \mathrm{~kJ} / \mathrm{mol}$. 另外, 该研究提出了一个可 以快速估计大量 MOFs 储氢性能的笁选参数——结合 率, 指 $\mathrm{MOF}$ 单晶胞中 $\mathrm{H}_{2}$ 强相互作用区域所占的体积比, 结合率越高, $\mathrm{H}_{2}$ 储存容量越大. 使用结合率作为评价指 标可以迅速地评估数据库中前 $20 \%$ ～30\%的优异候选材 料, 还能够大大降低计算成本. Goldsmith 等 ${ }^{[43]}$ 对从 CSD 数据库中提取的 4026 个 MOFs 进行储氢性能的计算分 析. 研究发现当 MOFs 的比表面积为 $3100 \sim 4800 \mathrm{~m}^{2} / \mathrm{g}$ 时, $\mathrm{H}_{2}$ 的单位体积容量达到最高 $(50 \sim 64 \mathrm{~g} / \mathrm{L})$, 然而继续 增大比表面积并不会促进储氢量升高. 此外, $\mathrm{H}_{2}$ 的单位 质量容量与单位体积容量呈现一个凹面的关系(图 9).

储氢 MOFs 的高通量计算篎选研究大多集中在极低 温 $(77 \mathrm{~K})$ 条件下的 $\mathrm{H}_{2}$ 储存. 近年来, 近室温条件下 MOFs 的储氢性能也有相关报道. 2014 年, Colón 等 ${ }^{[23]}$ 对 18383 个 hMOFs 和多孔芳香骨架(Porous Aromatic Frameworks, PAFs) 结构在 243 K/100-2 bar (吸附 100 bar, 脱附 2 bar)的 $\mathrm{H}_{2}$ 储运性能进行了基于 GCMC 的高通量 计算篎选. 结果发现其中 $\mathrm{H}_{2}$ 的最高质量吸附量和输运 量分别为 $9.35 \mathrm{wt} \%$ 和 $9.12 \mathrm{wt} \%$ (吸附热为 $3.2 \mathrm{~kJ} / \mathrm{mol}$ ), 最大体积吸附量和输运量分别为 $51 \mathrm{~g} / \mathrm{L}$ 和 $30 \mathrm{~g} / \mathrm{L}$ (吸附 热为 $6 \mathrm{~kJ} / \mathrm{mol}$ ). 值得关注的是, 作者认为材料中的有机 配体不同程度上进行 $\mathrm{Mg}$ 原子的醇化后可以改变材料的

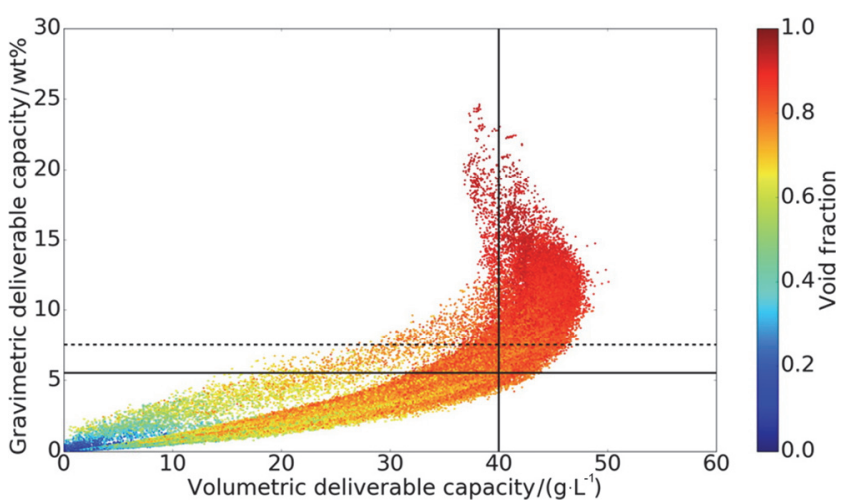

图 $8 \mathrm{H}_{2}$ 的质量输运容量 $(w t \%)$ 与体积输运容量 $(\mathrm{g} / \mathrm{L})$ 关系图. 黑色实 线表示 2015 年 DOE 储氢目标 $(5.5 \mathrm{wt} \%$ 和 $40 \mathrm{~g} / \mathrm{L})$, 虚线黑线表示 DOE 最终储氢目标 $(7.5 \% \mathrm{wt} \%$ 和 $70 \mathrm{~g} / \mathrm{L})$. 这些线的右上区域所含的 MOFs 代表最有前景的候选材料 ${ }^{[29]}$

Figure 8 Gravimetric deliverable hydrogen capacity (wt\%) versus volumetric deliverable capacity $(\mathrm{g} / \mathrm{L})$. The solid black lines indicate the $2015 \mathrm{DOE}$ targets of $5.5 \mathrm{wt} \%$ and $40 \mathrm{~g} / \mathrm{L}$. The dashed black line indicates the DOE full fleet target of $7.5 \mathrm{wt} \%$. MOFs above and to the right of these lines represent the most promising candidates. Each point represents one $\mathrm{MOF}^{[29]}$

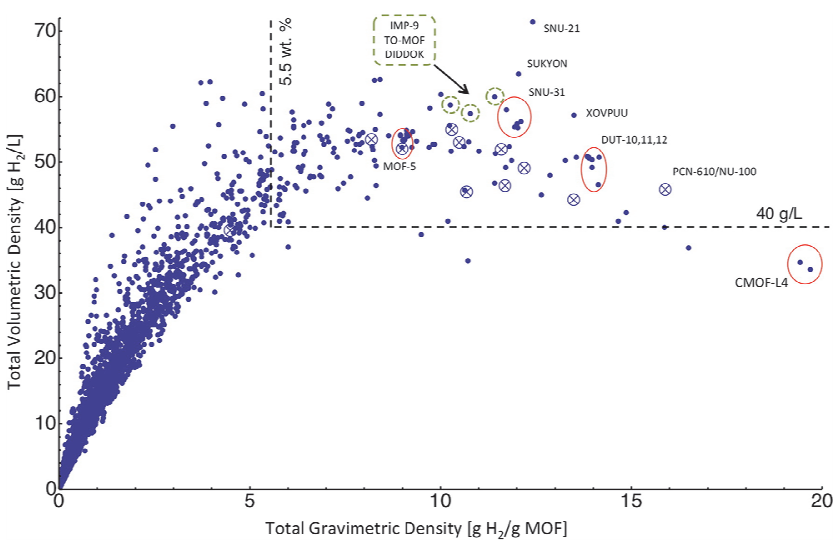

图 9 CSD 数据库中约 4000 种 MOFs 的理论储氢体积和重量密度(77 $\mathrm{K} / 35$ bar 下吸附 $+\mathrm{H}_{2}$ 气相). 展示的数据忽略系统的质量和体积贡献, 仅考虑 MOF 的质量和数量. 为了进行比较, 由虚线界定的区域代表 了 2017 年 DOE 储氢目标; 圆圈中表示在 CSD 数据库中常见的具有不 完整或无序晶体数据的 $\mathrm{MOFs}^{[43]}$

Figure 9 Theoretical total (adsorbed + gas phase $\mathrm{H}_{2}$ at $77 \mathrm{~K}$ and 35 bar) volumetric and gravimetric density of stored $\mathrm{H}_{2}$ in $\sim 4,000$ MOFs mined from the CSD. The data account only for the mass and volume of the MOF media; mass and volume contributions from the system are neglected. For comparison, the region bounded by the dashed lines represents the DOE 2017 targets for $\mathrm{H}_{2}$ storage systems. Crossed circles represent common MOFs with incomplete or disordered crystal data in the $\mathrm{CSD}^{[43]}$

吸附热，进而优化 $\mathrm{H}_{2}$ 的吸附能力. 构效关系分析发现， 为提高 $243 \mathrm{~K}$ 下 MOFs 的 $\mathrm{H}_{2}$ 质量输运能力, 应选择高空 隙率 $(>0.9)$ 和低 $\mathrm{Mg}$ 密度 $\left(0.5 \mathrm{mmol} / \mathrm{cm}^{3}\right)$. 基于 $\mathrm{cDFT}$ 的 储氢 MOFs 的高通量计算篎选也有报道. Fu 等 ${ }^{[30]}$ 研究采 用 cDFT 对 1200 个 hMOFs 在不同温度下的储氢性能进 行了模拟，并比较了基于四种函数的(MFA、FMSA、 WDA-Y 和 WDA-L) cDFT 与 GCMC 的模拟结果. 研究 发现 $243 \mathrm{~K} / 100 \mathrm{bar}$ 条件下基于四种函数的 cDFT 对 $\mathrm{H}_{2}$ 吸附量的预测都相当准确, 其中 FMSA 最为精确. 在 77 
$\mathrm{K} / 1$ bar 和 $77 \mathrm{~K} / 50$ bar 条件下发现基于 WDA 的 cDFT 方法最准确. 另外, cDFT 计算比传统的 GCMC 模拟方 法快至少一个数量级, 有效地降低了高通量计算篮选所 需的计算资源.

氢燃料动力汽车中的储氢系统通常需要高压低温 的储存条件, 例如丰田 Mirai14 ${ }^{[131]}$ 配有 $700 \mathrm{bar}$ 的压缩氢 气(Compressed Hydrogen Gas, CHG), 宝马公司也已经 在其概念 $i 8$ 汽车 ${ }^{[132]}$ 上尝试了 $350 \mathrm{bar}$ 的低温压缩 $\mathrm{H}_{2}$. 已 有研究表明基于多孔储氢材料的低温吸附式储氢系统 (罐体) 可以在 $77 \mathrm{~K} / 100 \mathrm{bar}$ 对氢气进行吸附储存, 在 160 $\mathrm{K} / 5$ bar 条件下进行解吸附 ${ }^{[133]}$. 为了开发在该操作条件 下最具储氢潜力的 MOFs, Gómez-Gualdrón 等 ${ }^{[49]}$ 基于 41 种不同的拓扑结构使用 “自顶至底” 的方法(图 2)构建 了 13512 个 hMOFs 结构, 并使用分子模拟方法计算 MOFs 在 $77 \mathrm{~K} / 100 \mathrm{bar}$ 和 $160 \mathrm{~K} / 5 \mathrm{bar}$ 的 $\mathrm{H}_{2}$ 输运容量. 结 果表明, 最大 $\mathrm{H}_{2}$ 吸附量(约为 $60 \mathrm{~g} / \mathrm{L}$ ) 出现在空隙率为 0.85 时; 在该操作条件下, 最优吸附热约为 $4 \mathrm{~kJ} / \mathrm{mol}$, 对 应的 $\mathrm{H}_{2}$ 输运容量为 $36 \sim 57 \mathrm{~g} / \mathrm{L}$. 研究还设计并制备了一 系列新型的 MOFs (she-MOF-x), 并测定了 she-MOF-1 (she-MOF-x 系列中体积吸附量最高)和 NU-1103 (先前 合成的具有优异 $\mathrm{H}_{2}$ 质量输运能力的 $M O F s$ ) 的 $\mathrm{H}_{2}$ 吸附量, 发现 NU-1103 比 she-MOF-1 更稳定, 且体积和质量输运 能力分别达到 $43.2 \mathrm{~g} / \mathrm{L}$ 和 $12.6 \mathrm{wt} \%$. 此外, Borboudakis 等 ${ }^{[112]}$ 采用机器学习算法准确预测了 100 种 eMOFs 在 77 $\mathrm{K} / 1$ bar 下的 $\mathrm{H}_{2}$ 吸附性能, 并与实验结果进行比较, 发 现机器学习预测的准确度与样本数量成正比.

\section{$4.3 \mathrm{CO}_{2}$ 捕捉}

随着温室效应日益严重, 高效低成本地降低 $\mathrm{CO}_{2}$ 的 排放成为全球研究热点. 目前分离捕获 $\mathrm{CO}_{2}$ 最有效的溶 剂分离法 ${ }^{[134]}$ 因其能耗大和有机溶剂造成的环境污染等 因素限制了其应用. 吸附分离法具有能耗低、清洁无污 染的优点, 其中 MOFs 材料在 $\mathrm{CO}_{2}$ 分离中表现出了良好 的性能. 迄今, 很多研究采用高通量计算笁选方法开发 具有优异性能的 MOFs 材料用于烟道气的分离 $\left(\mathrm{CO}_{2} / \mathrm{N}_{2}\right)$ 、天然气的净化 $\left(\mathrm{CO}_{2} / \mathrm{CH}_{4} / \mathrm{N}_{2}\right)$ 和预燃烧过程的 气体分离 $\left(\mathrm{CO}_{2} / \mathrm{H}_{2}\right)$ 等 $\mathrm{CO}_{2}$ 捕捉相关的气体分离.

2012 年, Watanabe 等 ${ }^{[39]}$ 采用高通量计算笁选研究了 CSD 数据库中 1163 种 MOFs 的 $\mathrm{CO}_{2} / \mathrm{N}_{2}$ 分离性能. 通过 GCMC 模拟发现在 $303 \mathrm{~K}$ 下 ZIF-90、OFERUN、 QERWAL 和 ZIF-65 表现出了良好的 $\mathrm{CO}_{2}$ 吸附能力(亨利 系数 $\left.K_{\mathrm{H}}>10 \mathrm{mmol} / \mathrm{g}\right)$. 进一步, 采用 $\mathrm{MD}$ 模拟研究 $\mathrm{CO}_{2}$ 在 MOFs 材料中的扩散、渗透和选择性，发现大约 $90 \%$ 的 MOFs 具有小于 400 的吸附选择性, 少量 MOFs 的吸 附选择性大于 1000 . 同时, 运用理想吸附溶液理论 (IAST) 研究在 $303 \mathrm{~K}$ 条件下 MOFs 在混合气氛 $\left(\mathrm{CO}_{2} / \mathrm{N}_{2}\right.$ $=0.15 / 0.85, V / V)$ 中的吸附情况. 其中, NOQLOV 表现出 了极佳的选择吸附性. 该研究表明了高通量计算篮选在 探究 MOFs 的 $\mathrm{CO}_{2} / \mathrm{N}_{2}$ 分离性能中的可行性. 此外, 在
$\mathrm{CO}_{2}$ 捕捉过程中, $\mathrm{H}_{2} \mathrm{O}$ 分子会与 $\mathrm{CO}_{2}$ 竞争吸附位点, 而 在 $\mathrm{CO}_{2}$ 捕捉前进行除湿增加了成本和操作的复杂性，因 此, $\mathrm{Li}$ 等 ${ }^{[99]}$ 对包含 5109 个 MOFs 的 CoRE MOF 数据库 进行了基于亨利常数 $\left(\mathrm{CO}_{2}\right.$ 和 $\mathrm{H}_{2} \mathrm{O}$ 亨利常数)的高通量计 算篮选，寻找在潮湿条件下有较高 $\mathrm{CO}_{2}$ 选择性的 MOFs. 研究篎选出了 15 种具有较高 $\mathrm{CO}_{2} / \mathrm{H}_{2} \mathrm{O}$ 选择性的 MOFs, 并在相对湿度(Relative Humidity, RH) 为 $80 \%$ 的条件下 利用 GCMC 模拟计算了三元组分 $\left(\mathrm{CO}_{2} / \mathrm{H}_{2} \mathrm{O} / \mathrm{N}_{2}\right.$, 其中 $\mathrm{CO}_{2} / \mathrm{N}_{2}=1 / 9, V / V, 298 \mathrm{~K} / 1$ bar)中 $\mathrm{CO}_{2}$ 的选择性, 结果表 明 15 种高 $\mathrm{CO}_{2} / \mathrm{H}_{2} \mathrm{O}$ 选择性的 MOFs 在 $\mathrm{CO}_{2} / \mathrm{N}_{2}$ 分离中同 样表现良好. 研究还发现篮选出的 MOFs 对 $\mathrm{CO}_{2}$ 比 $\mathrm{N}_{2}$ 和 $\mathrm{H}_{2} \mathrm{O}$ 具有更强的相互作用. 同时, 范德华相互作用在 $\mathrm{CO}_{2}$ 和 $\mathrm{N}_{2}$ 吸附中发挥主导的作用, 而对于极性 $\mathrm{H}_{2} \mathrm{O}$ 分 子, 静电作用更为重要, 表明 $\mathrm{H}_{2} \mathrm{O}$ 的吸附模拟更需要精 确的 MOFs 点电荷. 为进一步选择适用于 $\mathrm{CO}_{2} / \mathrm{N}_{2}$ 分离的 MOFs 膜材料, Budhathoki 等 ${ }^{[117]}$ 采用分子动力学模拟和 Maxwell model 对基于 112888 个 eMOF/hMOFs 和 9 种 聚合物组成的约 100 万种混合基膜 (Mixed Matrix Membrane, MMM)的 $\mathrm{CO}_{2}$ 分离性能进行了评价. 研究发 现 LCD 在 $4 \sim 10 \AA$ 和 PLD 在 $4 \sim 5 \AA$ 的 MOFs 具有更 高的选择吸附性能, 而为了提高 $\mathrm{MMM}$ 的分离性能, 所 采用的 MOFs 的渗透性和选择性都要远高于聚合物本 身. 此外, 高通量计算篮选还对 10995 个 MTV-MOFs 的 $\mathrm{CO}_{2} / \mathrm{N}_{2}$ 的分离性能进行了 $\mathrm{GCMC}$ 模拟 ${ }^{[32]}$, 研究发现孔 径大小比官能团种类对 MTV-MOFs 的 $\mathrm{CO}_{2}$ 吸附和 $\mathrm{CO}_{2} / \mathrm{N}_{2}$ 的分离性能发挥更重要的作用.

为了寻找可用于天然气净化 $\left(\mathrm{CO}_{2} / \mathrm{N}_{2} / \mathrm{CH}_{4}\right.$ 分离 $)$ 的良 好 MOFs 吸附剂, Qiao 等 ${ }^{[102]}$ 对 $\mathrm{hMOFs}$ 数据库中的 137953 个 hMOFs 进行了基于 GCMC 和 MD 的膜分离 高通量篎选. 研究初步篎选出了 17257 个PLD在 $3 \sim 4 \AA$ 之间的 MOFs, 之后采用分子模拟分别计算了在 $\mathrm{CO}_{2}$ 、 $\mathrm{N}_{2}$ 和 $\mathrm{CH}_{4}$ 的亨利常数和扩散系数. 对于 $\mathrm{CO}_{2} / \mathrm{CH}_{4}$ 分离, 根据选择渗透率大于 100 和 $\mathrm{CO}_{2}$ 的渗透率大于 104 barrer, 笁选出 328 个 MOFs. 在 $\mathrm{N}_{2} / \mathrm{CH}_{4}$ 分离中, 有 30 个 MOFs 满足选择渗透率大于 10 且 $\mathrm{N}_{2}$ 的渗透率大于 50 barrer. 选择上述两组中重叠的 24 个 MOFs 进一步研究 其在三元组分 $\left(\mathrm{CO}_{2} / \mathrm{N}_{2} / \mathrm{CH}_{4}=0.1 / 0.7 / 0.2, V / V / V\right)$ 的分离 性能. 在 $298 \mathrm{~K}$ 和 $10 \mathrm{bar}$ 条件下, 对 24 个 MOFs 进行吸 附、扩散和渗透的评估, 最终确定了 5 个具有最优异分 离性能的 MOFs, 其 PLD 为 $3.0 \sim 3.16 \AA$, 孔径分布 $\mathrm{PSD} \%{ }_{(3-3.2 \AA)}$ 为 $33 \% \sim 42 \%$. 此外, 研究还发现吸附和扩 散都对 $\mathrm{CO}_{2} / \mathrm{CH}_{4}$ 分离有积极作用, 而 $\mathrm{N}_{2} / \mathrm{CH}_{4}$ 的分离主 要是扩散作用驱动的. 之后，他们 ${ }^{[118]}$ 还采用主成分分 析(Principal Component Analysis, PCA)、MLR 和 DT 算 法对 4764 种 eMOFs 的 $\mathrm{CO}_{2} / \mathrm{N}_{2} / \mathrm{CH}_{4}$ 结构与其膜分离性 能之间的构效关系进行了描述.

Wilmer 等 ${ }^{[90]}$ 对 137953 个 hMOFs 进行分子模拟探 究其 $\mathrm{CO}_{2}$ 分离性能及其构效关系(图 10). 通过变压吸附 
(Pressure-swing Adsorption, PSA, $P^{\text {ads }}=5$ bar, $P^{\text {des }}=1$ bar) 和真空变压吸附(Vacuum-swing Adsorption, VSA, $P^{\mathrm{ads}}=$ 1 bar, $P^{\text {des }}=0.1$ bar)方法研究了 MOFs 对天然气 $\left(\mathrm{CO}_{2} / \mathrm{CH}_{4}=10 / 90, \mathrm{~V} / \mathrm{V}\right)$ 、沼气 $\left(\mathrm{CO}_{2} / \mathrm{CH}_{4}=50 / 50, \mathrm{~V} / \mathrm{V}\right)$ 和燃 煤废气 $\left(\mathrm{CO}_{2} / \mathrm{N}_{2}=10 / 90, V / V\right)$ 的 $\mathrm{CO}_{2}$ 吸附分离性能. 研究 采用五种评价标准: $\mathrm{CO}_{2}$ 吸附量、 $\mathrm{CO}_{2}$ 工作吸附量、 $\mathrm{CO}_{2}$ 选择性、可再生性能以及选择系数对 MOFs 的 $\mathrm{CO}_{2}$ 分离 吸附性能进行综合评价. 构效关系分析揭示了 MOFs 的 结构特征(即空隙率、孔径大小和比表面积)、 $\mathrm{CO}_{2}$ 吸附 热 $\left(Q_{\mathrm{st}}\right)$ 、化学特征(即官能团种类)与 $\mathrm{MOF}$ 的 $\mathrm{CO}_{2}$ 分离 性能之间的相互关系, 对高性能 MOFs 的理性设计与合 成具有指导意义. $\mathrm{Li}$ 等 ${ }^{[44]}$ 采用 GCMC 方法研究了具有拓 扑多样性的 151 种 $\mathrm{eMOFs}$ 在变温吸附 (Temperature-swing Adsorption, TSA)过程中(温度由 323 $\mathrm{K}$ 变化到 $423 \mathrm{~K}$ )对 $\mathrm{CO}_{2} / \mathrm{CH}_{4}$ 的分离性能. 他们提出了一 种新的表征吸附能量密度的参数——吸附度 (Adsorbility, AD), 定义为吸附剂的吸附热与空隙率的比 值. 研究以 MOFs 的可再生性能为主并结合三种常用的 评价指标 $\left(\mathrm{CO}_{2}\right.$ 吸附量、吸附选择性和再生性能)来探究 MOFs 材料与 $\mathrm{CO}_{2} / \mathrm{CH}_{4}$ 分离体系的构效关系, 结果表明 四个评价标准与 $\mathrm{MOF}$ 的 $\mathrm{CO}_{2}$ 和 $\mathrm{CH}_{4}$ 的吸附度差 $(\Delta \mathrm{AD})$ 表现出紧密相关性, 其中可再生性随着 $\triangle \mathrm{AD}$ 的增大出 现峰值. 通过篎选, 确定了 Cu-TDPAT 和 IRMOF-1-2L 是 TSA 过程中具有优异 $\mathrm{CO}_{2} / \mathrm{CH}_{4}$ 分离性能的 MOFs, 而 具有更高热稳定性和水稳定性的 $\mathrm{Cu}$-TDPAT 被认为是最 佳的候选材料. Aghaji 等 ${ }^{[105]}$ 对 324500 个 hMOFs 在传统 的天然气净化的条件下 $(298 \mathrm{~K} / 10-1 \mathrm{bar})$ 进行了定量构效 关系(QSPR)分析. 基于孔径、空隙率和表面积的计算, 采用决策树 DT 模型发现当空隙率低于 0.27 、最大孔径 小于 $6.6 \AA$ 时, MOFs 的 $\mathrm{CO}_{2} / \mathrm{CH}_{4}$ 选择性高于 10 ; 当孔径 小于 $8.5 \AA$ 、比表面积大于 $2300 \mathrm{~m}^{2} / \mathrm{g}$ 时, MOFs 的 $\mathrm{CO}_{2}$
吸附工作能力高于 $4 \mathrm{mmol} / \mathrm{g}$.

基于机器学习(ML)的高通量计算笁选也用于篎选 具有高效 $\mathrm{CO}_{2}$ 分离性能的 MOFs. 基于 ML 算法的高通 量篎选可以使篎选时间减少一个数量级, 实现快速高效 识别具有优异 $\mathrm{CO}_{2}$ 捕捉性能的 MOFs 的目标. Fernandez 等 ${ }^{[47]}$ 利用 SVMs 算法对 324500 个 $\mathrm{hMOFs}$ 进行 $\mathrm{CO}_{2}$ 捕 捉性能的评价. 其中 $10 \%$ 的 MOFs (32450个 MOFs) 作为 训练集, 剩余的 292050 个 MOFs 作为测试集用来验证构 效关系预测的可靠性. 研究发现提取的构效关系模型可 以准确发现 “测试集” 中 $\mathrm{CO}_{2}$ 吸附性能在前 1000 的 MOFs 中的 945 个 MOFs, 准确率达 95\%. Aghaji 和 Fernandez 等 ${ }^{[105]}$ 采用类似基于 SVMs 的方法，以 32500 个 hMOFs 作为训练集, 预测了 290000 个 hMOFs 的 $\mathrm{CO}_{2} / \mathrm{CH}_{4}$ 分离性能, 其准确率达 $90 \%$. 相比其他 $\mathrm{ML}$ 算 法, 基因遗传算法(GA)适用于从大型数据库中快速搜 索性能优异的 MOFs. Chung 等 ${ }^{[107]}$ 采用 GA 算法从55163 个 $\mathrm{hMOFs}$ 中篮选出了用于 $\mathrm{CO}_{2} / \mathrm{H}_{2}$ 分离的高性能吸附剂. 研究通过三个独立的 GA 循环以优化不同的评价标准: $\mathrm{CO}_{2}$ 工作吸附量 $\left(\Delta \mathrm{N}_{1}\right) 、 \mathrm{CO}_{2} / \mathrm{H}_{2}$ 的选择性 $\left(\alpha^{\mathrm{ads}}{ }_{12}\right)$ 以及吸附 剂性能(Adsorbent Performance Score, APS)即前两者的 乘积. 随着 GA 的运行, 每一代 MOFs 的性能都得到提 高, 运行 10 代后, 最佳 $\mathrm{CO}_{2}$ 工作能力从约 $7 \mathrm{~mol} / \mathrm{kg}$ 提高 到 $8 \mathrm{~mol} / \mathrm{kg}, \mathrm{CO}_{2} / \mathrm{H}_{2}$ 的选择性从 700 提高到 2600 , 吸附 剂性能从约 $1000 \mathrm{~mol} / \mathrm{kg}$ 提高到 $1200 \mathrm{~mol} / \mathrm{kg}$. 与 $\mathrm{GCMC}$ 模拟相比, GA 算法效率更高, 将计算时间减少两个数 量级. 篮选出的高效 MOF NOTT-101/OEt 的 $\mathrm{CO}_{2}$ 的工作 吸附量为 $3.8 \mathrm{~mol} / \mathrm{kg}, \mathrm{CO}_{2} / \mathrm{H}_{2}$ 选择性为 60 , 是所有公开 报道的相同条件下 $\mathrm{CO}_{2} / \mathrm{H}_{2}$ 分离性能最好的材料. 此外, Anderson 等 ${ }^{[119]}$ 采用 DFT、GCMC 和机器学习算法阐明 了机器学习算法可用于准确揭示官能团修饰和拓扑结 构对 hMOFs (约 400 种)的 $\mathrm{CO}_{2}$ 吸附性能的影响规律.
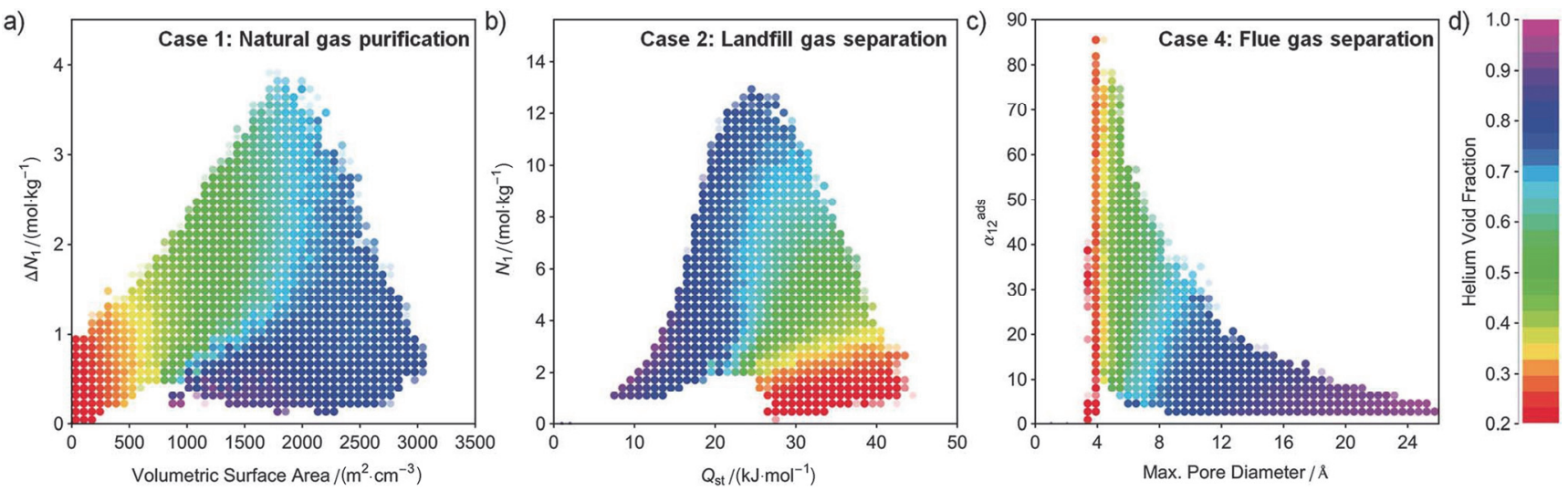

图 10130000 多种 hMOFs 的 $\mathrm{CO}_{2}, \mathrm{CH}_{4}$ 和 $\mathrm{N}_{2}$ 吸附的构效关系. (a) $\mathrm{CO}_{2}$ 工作容量 $\left(\Delta N_{1}\right)$ 和单位体积表面积, (b) 2.5 bar 下的 $\mathrm{CO}_{2}$ 吸附量 $\left(N_{1}\right)$ 和 $\mathrm{CO}_{2}$ 吸附热 $\left(Q_{\mathrm{st}}\right)$ 以及 $(\mathrm{c}) \mathrm{CO}_{2} / \mathrm{N}_{2}$ 的选择性 $\left(\alpha^{\mathrm{ads}}{ }_{12}\right)$ 和最大孔径之间存在的构效关系. $Q_{\mathrm{st}}$ 由最低模拟压力 $(0.01 \mathrm{bar})$ 下的 $\mathrm{CO}_{2}$ 吸附确定的. 如果存在超过 25 个结构, 则每幅图被划分为 $50 \times 50$ 个区域, 这些区域由实心圆表示. 实心圆的颜色代表绘图区域中所有结构(d)氦空隙率的平均值 ${ }^{104]}$

Figure 10 A sample of structure-property relationships derived from simulated $\mathrm{CO}_{2}, \mathrm{CH}_{4}$, and $\mathrm{N}_{2}$ adsorption in over 130000 hypothetical MOFs. Clear relationships can be discerned between (a) $\mathrm{CO}_{2}$ working capacity $\left(\Delta N_{1}\right)$ and surface area, (b) $\mathrm{CO}_{2}$ uptake $\left(N_{1}\right)$ at 2.5 bar and $\mathrm{CO}_{2}$ heat of adsorption $\left(Q_{\mathrm{st}}\right)$, and (c) selectivity of $\mathrm{CO}_{2}$ over $\mathrm{N}_{2}\left(\alpha^{\text {ads }}{ }_{12}\right)$ and maximum pore diameter. $Q_{\mathrm{st}}$ values are determined from $\mathrm{CO}_{2}$ adsorption at the lowest simulated pressure: 0.01 bar. Each plot is divided into $50 \times 50$ regions that are represented by a filled circle if more than 25 structures exist within the region. The color of each circle represents the average (d) helium void fraction of all structures in that plot region ${ }^{[104]}$ 


\section{4 能源气体分离}

高通量计算篎选在开发高效能源气体分离领域也 有广泛的应用, 主要包含 $\mathrm{H}_{2}$ 净化 $\left(\mathrm{CH}_{4} / \mathrm{H}_{2}\right), \mathrm{CH}_{4}$ 净化和 烃类的分离等. 使用 MOFs 实现高效低能耗的 $\mathrm{H}_{2}$ 净化 也成为近年来研究的热点之一. 2012 年, $\mathrm{Wu}$ 等 ${ }^{[33]}$ 采用基 于 GCMC 的高通量计算篮选方法研究了 105 种 MOFs 在 PSA 过程中对 $\mathrm{CH}_{4} / \mathrm{H}_{2}$ 等量混合气体的分离性能. 研 究发现 MOF-604、CPL-2 和 ZIF-11 等在低压条件 $(0.05$ $0.3 \mathrm{MPa}$ ), MOF-604、MIL-102 和 IRMOF-20 等在相对高 压条件下 $(0.1 \sim 2 \mathrm{MPa})$ 都具有比传统沸石和碳材料更优 的 $\mathrm{CH}_{4} / \mathrm{H}_{2}$ 分离性能. Altintas 等 ${ }^{[116]}$ 采用 GCMC 模拟探究 了 CSD 数据库中的 4350 个 MOFs 在 $298 \mathrm{~K} / 10-1 \mathrm{bar}$ 条 件下的 $\mathrm{CH}_{4} / \mathrm{H}_{2}$ 分离性能. 如图 11 所示, 研究发现 QUQQID (APS $=802) 、 U T E X I B 、 P A R M I G$ 和 ADIQEL 等 MOFs 具有较高的 $\mathrm{CH}_{4} / \mathrm{H}_{2}$ 选择性, 分别达 255、1520、 51 和 $394, \mathrm{CH}_{4}$ 工作吸附量分别为 $3.15 \mathrm{~mol} / \mathrm{kg} 、 0.43$ $\mathrm{mol} / \mathrm{kg} 、 1.04 \mathrm{~mol} / \mathrm{kg}$ 和 $1.30 \mathrm{~mol} / \mathrm{kg}$. 可再生性能是评价 PSA 分离性能的另一个重要指标, 研究发现可再生性能 $R \%>85 \%$ 的有 1101 种 $\mathrm{MOFs}$, 其金属团簇含 $\mathrm{Zn}(33 \%)$ 、 $\mathrm{Cu}(16 \%)$ 和 $\mathrm{Co}(11 \%)$ 的 MOFs 最为常见.

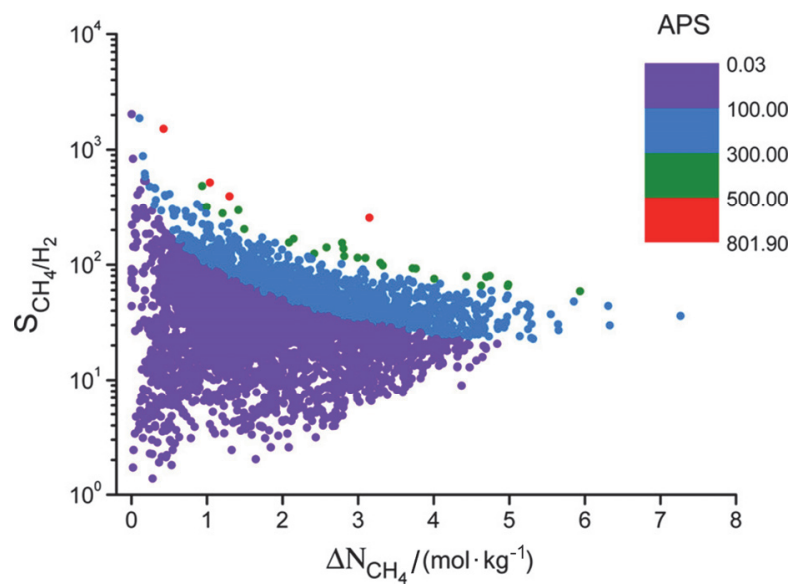

图 $11 \mathrm{MOFs}$ 的选择性和工作吸附量之间的关系, 颜色由 APS 数值决 定. 显示红色的 MOFs (QUQQID、UTEXIB、PARMIG 和 ADIQEL) 是最具应用前景的 $M O F s^{[116]}$

Figure 11 Selectivities and working capacities of MOFs color-coded according to their APSs. Exceptionally promising MOFs shown with red colors are QUQQID, UTEXIB, PARMIG and ADIQEL ${ }^{[116]}$

除了使用传统的 GCMC 模拟之外, Guo 等 ${ }^{[98]}$ 采用 cDFT 方法探究了 1200 个 hMOF 的 $\mathrm{CH}_{4} / \mathrm{H}_{2}$ 的分离性能. 通过模拟 $298 \mathrm{~K} / 0.75 \mathrm{MPa}$ 和 $298 \mathrm{~K} / 4 \mathrm{MPa}, \mathrm{CH}_{4} / \mathrm{H}_{2}=1 / 1$ $(V / V)$ 条件下 $\mathrm{CH}_{4} / \mathrm{H}_{2}$ 的选择性, 发现低压 $(0.75 \mathrm{MPa})$ 和高 压(4 MPa)下, MOFs 的 $\mathrm{CH}_{4} / \mathrm{H}_{2}$ 选择性最高为 150 和 70 . $303 \mathrm{~K}, \mathrm{CH}_{4} / \mathrm{H}_{2}=1 / 10(V / V)$ 条件下 MOFs 的最高选择性 为 165 , 因此认为低压和低 $\mathrm{CH}_{4}$ 浓度下更适合使用 MOFs 进行分离. 同时, 研究发现选择性是由 MOFs 的 结构特征决定的, 当 MOFs 的孔径恰好适合容纳 1 个 $\mathrm{CH}_{4}$ 分子时, 其选择性最高; 当相邻孔之间的距离约为
相邻 $\mathrm{CH}_{4}$ 分子的吸引距离(4 6 $\AA$ )时，由于气体分子之 间的相互作用, 使 MOFs 更有利于吸附 $\mathrm{CH}_{4}$, 可促进选 择吸附性. 另外, 天然气中除了 $\mathrm{CO}_{2}$ 和 $\mathrm{N}_{2}$ 杂质气体之 外, 叔丁基硫醇(tert-butyl mercaptan, TBM)也是输运天 然气管道中的含硫杂质气体. 天然气中 TBM 的燃烧会 产生有害的 $\mathrm{SO}_{x}$ 并腐蚀浴轮, 因此, Nazarian 等 ${ }^{[101]}$ 采用 基于 GCMC 的高通量篎选对 4519 个 CoRE MOF 的 $\mathrm{CH}_{4} / \mathrm{TBM}$ 的分离性能进行了篮选. 首先通过亨利系数 计算的 $\mathrm{CH}_{4} / \mathrm{TBM}$ 的选择性选取 1497 种 $\mathrm{CH}_{4} / \mathrm{TBM}$ 选择 性大于 103 、吸附热小于 $70 \mathrm{~kJ} / \mathrm{mol}$ 且不含镧系金属的 MOFs 进行 GCMC 模拟. 结果发现 354 个 MOFs 具有大 于 $200 \mathrm{mg} / \mathrm{g}$ 的 TBM 吸附量. 其中, MIL-53 和 Cu-BTC 具有高 TBM 选择性但结构不够稳定; 而篮选的 BIBHUX 和 MFU-4 是在 $\mathrm{CH}_{4} / \mathrm{TBM}$ 分离过程中兼具优异 选择性和水稳定性的 MOFs. 高通量计算篮选在 MOFs 的 $\mathrm{C}_{2} \mathrm{H}_{6} / \mathrm{C}_{2} \mathrm{H}_{4}$ 分离领域也具有一定的研究. Altintas 等 ${ }^{[103]}$ 对 CCDC 数据库中的 278 种 eMOFs 进行 $\mathrm{C}_{2} \mathrm{H}_{6} / \mathrm{C}_{2} \mathrm{H}_{4}$ 和 $\mathrm{C}_{2} \mathrm{H}_{6} / \mathrm{CH}_{4}$ 分离性能的 GCMC 模拟. 结果表明大部分 MOFs 的 $\mathrm{C}_{2} \mathrm{H}_{6} / \mathrm{C}_{2} \mathrm{H}_{4}$ 分离性能比传统沸石更高, 其 $\mathrm{C}_{2} \mathrm{H}_{6}$ 的工作吸附量超过 $3 \mathrm{~mol} / \mathrm{kg}$, 远高于沸石中的 1.75 $\mathrm{mol} / \mathrm{kg}$. 构效关系分析还发现 $\mathrm{C}_{2} \mathrm{H}_{6} / \mathrm{C}_{2} \mathrm{H}_{4}$ 和 $\mathrm{C}_{2} \mathrm{H}_{6} / \mathrm{CH}_{4}$ 的 选择性随着压力的降低而提高, 随着孔径的增加而降 低.

\section{5 稀有气体分离}

稀有气体在工业、医学、科研等领域有着广泛的应 用. 纯的 $\mathrm{Xe}$ 和 $\mathrm{Kr}$ 在医疗(如麻醉、成像)和照明(如荧光 标志、机场跑道灯)领域有重要的应用. 工业上分离稀有 气体主要采用的是低温分馏空气法(获取 $\mathrm{Ne} 、 \mathrm{Ar} 、 \mathrm{Kr}$ 和 $\mathrm{Xe}$ )或低温分馏天然气法(获取 $\mathrm{He}$ ), 然而低温分馏法 耗能十分巨大，而室温吸附分离法能够有效降低能耗, 利用 MOFs 对稀有气体进行吸附分离是更具前景的技 术. 因此，采用高通量方法篎选出对稀有气体具有优良 吸附性能的 MOFs 具有重要意义 ${ }^{[135]} .2011$ 年, Ryan 等 ${ }^{[136]}$ 对 IRMOF-1、MOF-505 和 HKUST-1 等 8 种常见 且具有不同孔径的 MOFs 进行 GCMC 模拟, 发现孔径较 大 MOFs 的 $\mathrm{Xe} / \mathrm{Kr}$ 选择性较差, 而小孔径(可容纳一个 $\mathrm{Xe}$ 原子的尺寸)可以增强对 Xe 的吸附. 在研究的所有 MOFs 中, Pd-MOF 在较宽压力范围内 $(0.01 、 0.1$ 和 1 MPa)都具有最高的选择性. HKUST-1 在低压下选择性 较好, 在高压下由于大孔的吸附导致选择性急剧下降, MOF-505 具有两种不同类型的小孔, 在低压和和高压 下均能保持较高的选择性. Sikora 等 ${ }^{[63]}$ 对 hMOFs 数据库 中的 137953 种 MOFs 的 Xe/ $\mathrm{Kr}$ 分离性能进行了 GCMC 模拟. 如图 12 所示, 研究发现在 $5 \mathrm{bar}$ 条件下, 具有最高 $\mathrm{Xe} / \mathrm{Kr}$ 选择性的 MOFs 的 LCD 略大于 Xe 原子直径(约 $4 \sim 6 \AA$ ). 当 MOFs 的 $L C D / P L D$ 在 1 和 2 之间时, $X e / K r$ 选择性较高. 

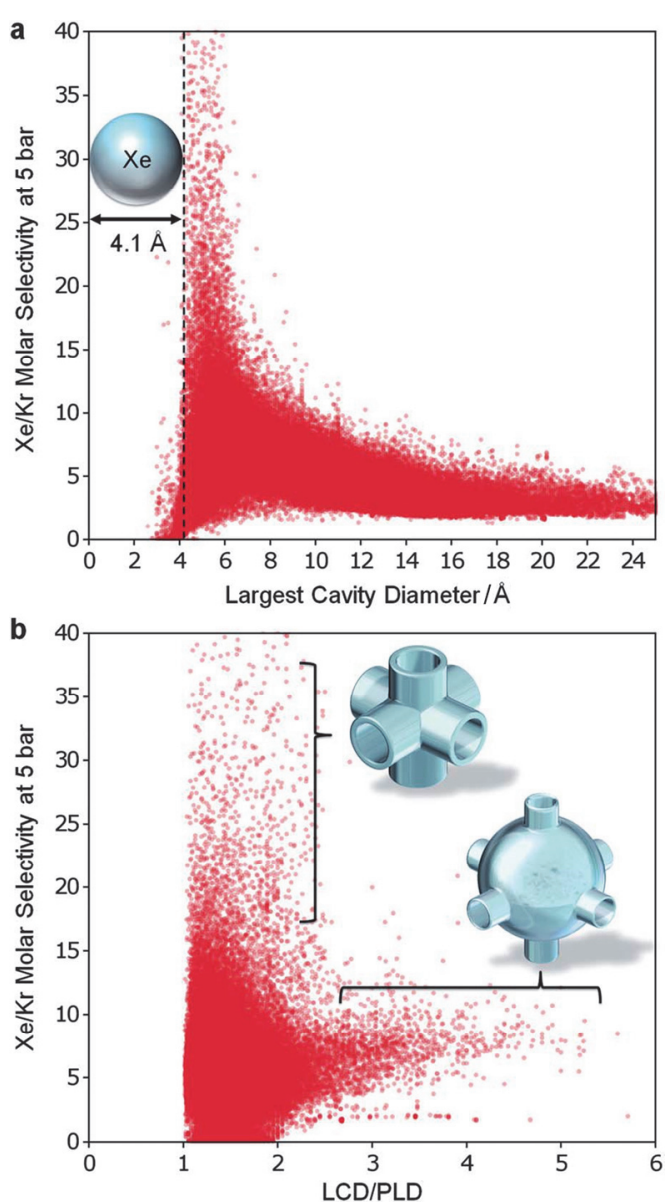

图 $12 \mathrm{Xe} / \mathrm{Kr}$ 分离性能的最优 MOFs 的几何特征 $(273 \mathrm{~K} / 5 \mathrm{bar}$ ). (a)所有 hMOFs 材料的最大 Xe/ Kr 摩尔选择性集中 LCD 略大于 Xe 原子直径 的区域(约 4 6 $\AA$ ); (b) 具有由狭窄通道连接的大孔(LCD/PLD 大于 2) 的 MOFs 的选择性较低, 孔形貌类似于宽度相同的管状结构 (LCD/PLD 在 1 和 2 之间)的 MOFs 具有较高的 $\mathrm{Xe} / \mathrm{Kr}$ 摩尔选择性. 为 了清晰表达, 两幅图中的最高选择性均截止在 $40^{[63]}$

Figure 12 The geometry of an optimal MOF for $\mathrm{Xe} / \mathrm{Kr}$ separation. (a) In all of the hypothetical MOFs, the highest selectivities were shared amongst the narrow subset of structures with LCD slightly greater than the diameter of a xenon atom. (b) The least selective MOFs had large cavities connected by narrow channels (LCD/PLD ratio greater than 2) while the best MOFs had tube-like pore morphologies (LCD/PLD ratio between 1 and 2). In both graphs, the selectivity has been cutoff at 40 for clarity ${ }^{[63]}$

为了寻找标准条件下在惰性混合气体中能够有效 分离 $\mathrm{Xe}$ 的 MOFs, Sumer 等 ${ }^{[35]}$ 采用 GCMC 和 $\mathrm{MD}$ 模拟 对 115 种 eMOFs 的 $\mathrm{Xe} / \mathrm{Kr} 、 \mathrm{Xe} / \mathrm{Ar}$ 和 $\mathrm{Xe} / \mathrm{Rn}$ 分离性能和 膜分离性能进行探究. 结果表明当 MOFs 的 LCD 在 4.3 6.8 $\AA$, 空隙率小于 0.58 , 质量比表面积在 $150 \sim$ $1000 \mathrm{~m}^{2} / \mathrm{g}$ 的范围内时, 其气体分离性能最好. 同时, 研 究发现了 $\mathrm{Xe} / \mathrm{Kr} 、 \mathrm{Xe} / \mathrm{Ar}$ 和 $\mathrm{Xe} / \mathrm{Rn}$ 分离性能前 10 的 MOFs, 其中 CAYGIU、EYOPOY、EYOPUE、EYOQAL、 NAZBAT 和 PAQFIY 是对三种混合气体都具有优异分 离性能的材料. 这些发现能够为设计和合成新的具有优 异惰性混合气体分离性能的 MOFs 提供指导. 另外, 利 用 MOFs 从天然气中吸附分离回收 $\mathrm{He}$ 是一种重要的技 术. Kadioglu 等 ${ }^{[13]}$ 率先对 MOFs 的 $\mathrm{He} / \mathrm{CH}_{4}$ 的分离性能 进行了大规模的计算篮选, 他们在 $\mathrm{He} / \mathrm{CH}_{4}$ 分离的工业
条件下 $(298 \mathrm{~K} / 0.01-10 \mathrm{bar})$, 结合 GCMC 和 MD 方法计 算膜分离的选择性和渗透率等. 研究发现 MOFs 具有较 强的 $\mathrm{CH}_{4}$ 吸附性, 而 $\mathrm{He}$ 则具有较高的扩散速率. 在所 研究的 139 种 MOFs 结构中, 仅 4 种 MOFs (GUPCUQ01、HUTZAX01、PEHBIO 和 BIYTEJ)实现对 $\mathrm{He}$ 的选择性吸附, 其他 MOFs 则是通过吸附 $\mathrm{CH}_{4}$ 实现 $\mathrm{CH}_{4} / \mathrm{He}$ 分离. 此外, PLD 在 3.8 4.0 $\AA$ 之间的 MOFs 膜 具有良好的 $\mathrm{He} / \mathrm{CH}_{4}$ 选择性. 尽管如此, 未来仍然需要 对大量 MOFs 进行篮选才能获得 MOFs 与 $\mathrm{He} / \mathrm{CH}_{4}$ 分离 性能之间更精确的构效关系模型.

\section{6 其他物质的吸附分离}

工业应用中, 除了 $4.1 \sim 4.5$ 节中所述常见气体的储 存、捕捉和分离应用，还存在一些对环境有污染或有毒 的废气需要处理, 例如脱硫、氨气吸附和甲醛吸附等. 使用 MOFs 作为吸附剂的吸附分离技术可以克服氢化脱 硫技术和传统吸附材料存在的不足, 因此, 近年来也陆 续出现有关高通量计算篮选用于废气吸附的 MOFs 的研 究.

二苯并噻吩(Dibenzothiophene, DBT)是柴油中相当 顽固的含硫化合物, 为了寻找高性能脱硫吸附剂, Liu 等 ${ }^{[96]}$ 通过 $\mathrm{cDFT}$ 方法对 1200 个 $\mathrm{hMOFs}^{[28]}$ 的 DBT 吸附性 能进行了高通量计算篮选, 并将其中 458 个 $\mathrm{Cu}$ 基 $\mathrm{MOFs}$ 的 DBT 吸附能力与 HKUST- $1^{[137]}$ 的吸附性能进行对比. 结果发现, 在低浓度 $(10 \mathrm{ppmw})$ 的条件下, 有 108 个 MOFs 的吸附量超过了相同条件下 HKUST-1 的吸附量 $(0.06 \mathrm{~mol} / \mathrm{L})$, 其最高吸附量为 $1.62 \mathrm{~mol} / \mathrm{L}$; 在高浓度 (1152 ppmw)条件下, 有 126 个 MOFs 的吸附量超过 HKUST-1, 其中最高吸附量 $(2.99 \mathrm{~mol} / \mathrm{L})$ 约为 HKUST-1 的 2 倍. 此外, 结合径向分布函数, 作者发现低浓度和 高浓度下具有最佳 DBT 吸附性能的 MOFs 的吸附机制 是不同的，在低浓度吸附中分层吸附占主导地位，而在 高浓度吸附中可视为自由体积的填充.

挥发性有毒工业化学品(Toxic Industrial Chemicals, TICs, 如 $\mathrm{NH}_{3} 、 \mathrm{NO}_{x}$ 和甲醛等)的吸附净化是 MOFs 另外 一个应用领域，具有比传统活性炭更高的吸附量. Moghadam 等 ${ }^{[100]}$ 根据水的亨利系数快速识别 hMOFs 的 亲疏水性, 并通过 GCMC 模拟评价 MOFs 在室温常压、 $\mathrm{RH}=80 \%$ 条件下对 $\mathrm{NH}_{3}$ 和 $\mathrm{CH}_{4}$ 的吸附选择能力(图 13). 研究发现过于亲水的 MOFs 会吸附过多的水, 导致对 TICs 几乎没有选择吸附性; 过于疏水的 MOFs 对 TICs 的亲和力较低, 选择性也较低. 因此, 具有中等疏水性 且孔径大小与 TICs 动力学直径相当的 MOFs 具有最高 的选择性. 此外, 研究还发现含有卤族元素的 MOFs 的 TICs 选择性最低, 表面有烷基修饰的 MOFs 选择性最 高，这些较大的官能团可以通过减小孔径和增加疏水性 来影响对 TICs 吸附选择性. 另外, Bian 等 ${ }^{[138]}$ 采用基于 GCMC 的高通量计算篮选对 CoRE MOF 数据库中 1600 多种 MOFs 的甲醛/空气分离性能进行了研究, 实验研 

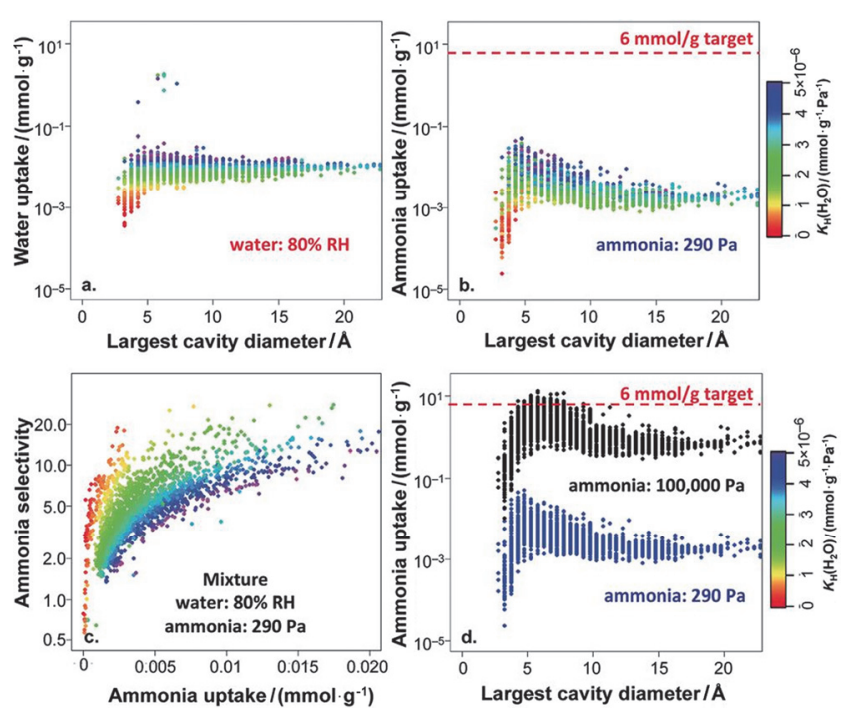

图 13 在 $298 \mathrm{~K}$ 对 2777 个未官能化疏水 MOFs 进行模拟的结果. (a) 纯组分水在 $80 \% \mathrm{RH}$, (b) 纯组分 $\mathrm{NH}_{3}$ 在 $290 \mathrm{~Pa}$ 条件下的模拟吸附量; (c)二元混合物 $\mathrm{NH}_{3}$ 在 $290 \mathrm{~Pa}$ 和水在 $80 \%$ 条件下的 $\mathrm{NH}_{3}$ 选择性; (d)纯 组分 $\mathrm{NH}_{3}$ 在 $290 \mathrm{~Pa}$ 和 $100000 \mathrm{~Pa}$ 条件下的模拟吸附量 ${ }^{[100]}$

Figure 13 Simulated adsorption amounts for (a) pure-component water at $80 \% \mathrm{RH}$, (b) pure-component ammonia at $290 \mathrm{~Pa}$, (c) selectivity of ammonia over water for a binary mixture of ammonia at $290 \mathrm{~Pa}$ and water $80 \% \mathrm{RH}$, (d) pure-component ammonia at $290 \mathrm{~Pa}$ and 100,000 Pa. All simulations were performed for 2777 unfunctionalized hydrophobic MOFs at $298 \mathrm{~K}^{[100]}$

究进一步验证了篮选出的 Y-BTC 的甲醛吸附量远高于 常规的 Cu-BTC, 是传统的活性炭的 5 倍. 此外, 空气中 还存在来自水果、蔬菜以及身体散发的少量臭味气体 ——硫醇(包括甲硫醇 $\mathrm{MeSH}$ 和乙硫醇 $\mathrm{EtSH}$ ). 使用多孔 材料进行硫醇吸附是一种经济高效的方法, Qiao 等 ${ }^{[50]}$ 通 过高通量䇥选研究了 $\mathrm{hMOFs}$ 和 CoRE MOF 数据库中共 142717 种 MOFs 的硫醇捕集性能, 模拟条件为 $298 \mathrm{~K} / 1$ bar、空气(78\%的 $\mathrm{N}_{2}$ 和 $22 \%$ 的 $\mathrm{O}_{2}$ ) 与 $10 \mathrm{ppm} \mathrm{MeSH}$ 和 10 ppm EtSH 的混合气体. 为了避免在吸附过程中水和硫 醇的竞争, 通过计算 MOFs 对水的亨利系数进行初步篮 选, 获得 31816 种疏水 MOFs (其中 31399 种来自 hMOFs 数据库, 417 种来自 CoER MOFs 数据库). GCMC 模拟结 果表明 MOFs 对硫醇的最高吸附量为 $70.86 \mathrm{mg} / \mathrm{g}$, 最高 选择性为 $2.6 \times 10^{7}$. 选择性较高的 MOFs 的空隙率为 $0.09 \sim 0.35$ 、吸附热为 $63 \sim 78 \mathrm{~kJ} / \mathrm{mol} 、 \mathrm{LCD}$ 为 $4.5 \sim 7.5$ $\AA$ 、体积比表面积约为 $80 \sim 640 \mathrm{~m}^{2} / \mathrm{cm}^{3}$. 此外, 通过主成 分分析(PCA)和多元线性回归模型(MLR)分析, 发现空 隙率、吸附热和体积比表面积在评估 MOFs 的硫醇吸附 性能中占主导地位, 特别是吸附热. 采用 DT 方法中发 现, 在吸附热 $>55 \mathrm{~kJ} / \mathrm{mol}$ 、空隙率 $<0.35$ 和体积比表面 积 $>270 \mathrm{~m}^{2} / \mathrm{cm}^{3}$ 的篎选策略中, 可以获得 $81.1 \%$ 具有目 标性能(硫醇吸附量 $>5 \mathrm{mg} / \mathrm{g}$, 选择性 $>15000$ ) 的 MOFs.

\section{5 总结与展望}

综上所述, MOFs 的高通量计算篮选是近十年来发
展起来的一种新的 MOFs 研发技术, 能够实现从大量 MOFs 材料中快速准确锁定高性能材料, 并结合实验进 行验证. 同时, 根据大量 MOFs 的结构特征与吸附性能 数据, 通过数学分析获得 MOFs 的构效关系模型, 从而 辅助新型高效 MOFs 的理性设计与应用. 尽管高通量计 算篎选在 MOFs 的研发领域已经获得飞速发展, 然而实 现 MOFs 的高效研发与商业化应用依然存在很多挑战. 首先, 在 MOFs 数据库方面, 由于高通量计算篎选中的 分子模拟所采用的 eMOFs 模型可能与实际结构存在差 距，因而导致预测结果的偏差. 而实验制备计算机构建 的高性能 hMOFs 可能困难重重, 因此, 将实验合成难度 作为一项评价标准纳入 hMOFs 的高通量计算篮选有望 在未来实现. 此外, 未来的高通量计算笁选还可能将 MOFs 的水/热稳定性和制备成本纳入评价标准，从而为 MOFs 的实际应用提供更多参考信息. 其次, 对于极性 气体分子, 比如水分子, 由于存在极强的相互作用而很 难在 GCMC 模拟中达到吸附平衡因而难以对 MOFs 的 水吸附性能进行高通量计算篮选, 未来高性能计算机的 发展可能解决这一难题. 最后, 尽管目前基于机器学习 算法的高通量计算篮选还未占据主流，未来越来越多 hMOFs 和 eMOFs 的出现将对计算资源有极高的需求, 机器学习必将成为未来高通量计算篮选的不可或缺的 重要方法.

\section{作者简介}

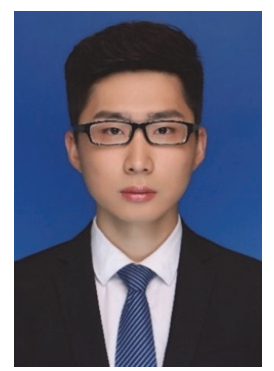

刘治鲁, 2018 年 6 月于东北大学获得学士学位, 2018 年 9 月至今在华中科技大学李松副教授课题组攻读硕士学位. 主 要研究兴趣为多孔材料的高通量计算篮选及其在能源储存转 化方面的应用.

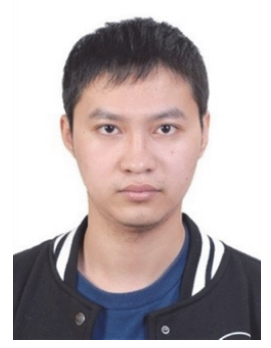

李炜, 2015 年于华中科技大学获得学士学位, 2015 年至今 师从李松副教授攻读博士学位。主要研究兴趣为高通量篮选 技术在多孔材料研究中的应用. 


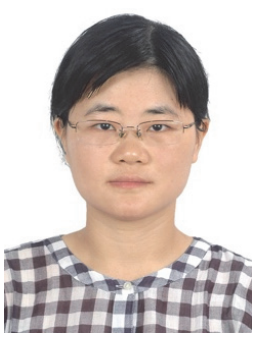

李松, 博士, 副教授. 2006 年和 2009 年自山东大学和韩国 成均馆大学获得学士和硕士学位. 2009 年至 2014 年在美国范 德堡大学化工系学习并获得博士学位. 2014 年至 2015 年 8 月 在美国西北大学化工系 Randall Q. Snurr 教授课题组进行博士 后研究. 2015 年 8 月加入华中科技大学能源与动力工程学院. 主要研究兴趣为金属有机骨架的高通量计算篎选及其在能源 储存转化和环境领域的应用.

\section{References}

[1] Li, H.; Eddaoudi, M.; O'Keeffe, M.; Yaghi, O. M. Nature 1999, 402, 276.

[2] O’Keeffe, M.; Peskov, M. A.; Ramsden, S. J.; Yaghi, O. M. Acc. Chem. Res. 2008, 41, 1782 .

[3] Férey, G. Chem. Soc. Rev. 2008, 37, 191.

[4] Horike, S.; Shimomura, S.; Kitagawa, S. Nat. Chem. 2009, 1, 695.

[5] Murray, L. J.; Dinca, M.; Long, J. R. Chem. Soc. Rev. 2009, 38, 1294.

[6] Sculley, J.; Yuan, D.; Zhou, H. C. Energy Environ. Sci. 2011, 4, 2721.

[7] Li, J. R.; Kuppler, R. J.; Zhou, H. C. Chem. Soc. Rev. 2009, 38, 1477.

[8] Verma, S.; Mishra, A. K.; Kumar, J. Acc. Chem. Res. 2010, 43, 79

[9] Li, J. R.; Sculley, J.; Zhou, H. C. Chem. Rev. 2012, 112, 869.

[10] Bae, Y. S.; Snurr, R. Q. Angew. Chem. 2011, 50, 11586.

[11] Allendorf, M. D.; Bauer, C. A.; Bhakta, R. K.; Houk, R. J. Chem. Soc. Rev. 2009, 38, 1330.

[12] Kreno, L. E.; Leong, K.; Farha, O. K.; Allendorf, M.; Van Duyne, R. P.; Hupp, J. T. Chem. Rev. 2012, 112, 1105.

[13] Horcajada, P.; Serre, C.; Vallet-Regí, M.; Sebban, M.; Taulelle, F.; Férey, G. Angew. Chem. 2006, 118, 6120 .

[14] Rocca, J. D.; Liu, D. M.; Lin, W. B. Acc. Chem. Res. 2011, 44, 957.

[15] Bernini, M. C.; Fairen-Jimenez, D.; Pasinetti, M.; Ramirez-Pastor, A. J.; Snurr, R. Q. J. Mater. Chem. B 2014, 2, 766.

[16] Kent, C. A.; Mehl, B. P.; Ma, L.; Papanikolas, J. M.; Meyer, T. J.; Lin, W. B. J. Am. Chem. Soc. 2010, 132, 12767.

[17] Kent, C. A.; Liu, D.; Ma, L.; Papanikolas, J. M.; Meyer, T. J.; Lin, W. B. J. Am. Chem. Soc. 2011, 133, 12940.

[18] Lee, C. Y.; Farha, O. K.; Hong, B. J.; Sarjeant, A. A.; Nguyen, S. T.; Hupp, J. T. J. Am. Chem. Soc. 2011, 133, 15858.

[19] Farrusseng, D.; Aguado, S.; Pinel, C. Angew. Chem. 2009, 48, 7502.

[20] Ma, L.; Abney, C.; Lin, W. B. Chem. Soc. Rev. 2009, 38, 1248.

[21] Lee, J.; Farha, O. K.; Roberts, J.; Scheidt, K. A.; Nguyen, S. T.; Hupp, J. T. Chem. Soc. Rev. 2009, 38, 1450.

[22] Farha, O. K.; Shultz, A. M.; Sarjeant, A. A.; Nguyen, S. T.; Hupp, J. T. J. Am. Chem. Soc. 2011, 133, 5652.

[23] Colón, Y. J.; Fairen-Jimenez, D.; Wilmer, C. E.; Snurr, R. Q. J. Phys. Chem. C 2014, 118, 5383.

[24] de Pablo, J. J.; Jones, B.; Kovacs, C. L.; Ozolins, V.; Ramirez, A. P. Curr. Opin. Solid State Mater. Sci. 2014, 18, 99.

[25] Jain, A.; Ong, S. P.; Hautier, G.; Chen, W.; Richards, W. D.; Dacek, S.; Cholia, S.; Gunter, D.; Skinner, D.; Ceder, G.; Persson, K. A. APL Mater. 2013, 1, 011002.

[26] Gomez-Gualdron, D. A.; Gutov, O. V.; Krungleviciute, V.; Borah, B.; Mondloch, J. E.; Hupp, J. T.; Yildirim, T.; Farha, O. K.; Snurr, R. Q. Chem. Mater. 2014, 26, 5632.

[27] Chung, Y. G.; Camp, J.; Haranczyk, M.; Sikora, B. J.; Bury, W.; Krungleviciute, V.; Yildirim, T.; Farha, O. K.; Sholl, D. S.; Snurr, R. Q. Chem. Mater. 2014, 26, 6185.

[28] Fu, J.; Tian, Y.; Wu, J. Z. AIChE J. 2015, 61, 3012.
[29] Bobbitt, N. S.; Chen, J.; Snurr, R. Q. J. Phys. Chem. C 2016, 120, 27328.

[30] Fu, J.; Liu, Y.; Tian, Y.; Wu, J. Z. J. Phys. Chem. C 2015, 119, 5374.

[31] Daff, T. D.; Woo, T. K. MRS Online Proc. Libr. 2014, 1523.

[32] Li, S.; Chung, Y. G.; Simon, C. M.; Snurr, R. Q. J. Phys. Chem. Lett. 2017, 8, 6135 .

[33] Wu, D.; Wang, C. C.; Liu, B.; Liu, D. H.; Yang, Q. Y.; Zhong, C. L. AIChE J. 2012, 58, 2078.

[34] Wilmer, C. E.; Leaf, M.; Lee, C. Y.; Farha, O. K.; Hauser, B. G.; Hupp, J. T.; Snurr, R. Q. Nat. Chem. 2011, 4, 83.

[35] Sumer, Z.; Keskin, S. Chem. Eng. Sci. 2017, 164, 108.

[36] Yang, W. Y.; Liang, H.; Qiao, Z. W. Acta Chim. Sinica 2018, 76, 785. (杨文远, 梁红, 乔智威, 化学学报, 2018, 76, 785.)

[37] Jiang, J. W. Curr. Opin. Chem. Eng. 2012, 1, 138.

[38] Moghadam, P. Z.; Li, A.; Wiggin, S. B.; Tao, A.; Maloney, A. G. P.; Wood, P. A.; Ward, S. C.; Fairen-Jimenez, D. Chem. Mater. 2017, $29,2618$.

[39] Watanabe, T.; Sholl, D. S. Langmuir 2012, 28, 14114.

[40] Allen, F. H. Acta Crystallogr. Sect. B: Struct. Sci. 2002, 58, 380.

[41] Willems, T. F.; Rycroft, C. H.; Kazi, M.; Meza, J. C.; Haranczyk, M. Microporous Mesoporous Mater. 2012, 149, 134.

[42] Hoshen, J.; Kopelman, R. Phys. Rev. B 1976, 14, 3438.

[43] Goldsmith, J.; Wong-Foy, A. G.; Cafarella, M. J.; Siegel, D. J. Chem. Mater. 2013, 25, 3373.

[44] Li, Z. J.; Xiao, G.; Yang, Q. Y.; Xiao, Y. L.; Zhong, C. L. Chem. Eng. Sci. 2014, 120, 59.

[45] The Computation-Ready, Experimental (CoRE) Metal-Organic Frameworks Database, http://gregchung.github.io/CoRE-MOFs/.

[46] Lin, L. C.; Berger, A. H.; Martin, R. L.; Kim, J.; Swisher, J. A.; Jariwala, K.; Rycroft, C. H.; Bhown, A. S.; Deem, M. W.; Haranczyk, M.; Smit, B. Nat. Mater. 2012, 11, 633.

[47] Fernandez, M.; Boyd, P. G.; Daff, T. D.; Aghaji, M. Z.; Woo, T. K. J. Phys. Chem. Lett. 2014, 5, 3056.

[48] McDaniel, J. G.; Li, S.; Tylianakis, E.; Snurr, R. Q.; Schmidt, J. R. J. Phys. Chem. C 2015, 119, 3143.

[49] Gómez-Gualdrón, D. A.; Colón, Y. J.; Zhang, X.; Wang, T. C.; Chen, Y.-S.; Hupp, J. T.; Yildirim, T.; Farha, O. K.; Zhang, J.; Snurr, R. Q. Energy Environ. Sci. 2016, 9, 3279.

[50] Qiao, Z. W.; Xu, Q. S.; Cheetham, A. K.; Jiang, J. W. J. Phys. Chem. $C$ 2017, 121, 22208.

[51] Qiao, Z. W.; Xu, Q. S.; Jiang, J. W. J. Mater. Chem. A 2018, 6, 18898 .

[52] Baburin, I. A.; Leoni, S. CrystEngComm 2010, 12, 2809.

[53] Hayashi, H.; Côté, A. P.; Furukawa, H.; O’Keeffe, M.; Yaghi, O. M. Nat. Mater. 2007, 6, 501.

[54] Lewis, D. W.; Ruiz-Salvador, A. R.; Gómez, A.; Rodriguez-Albelo, L. M.; Coudert, F.-X.; Slater, B.; Cheetham, A. K.; MellotDraznieks, C. CrystEngComm 2009, 11, 2272.

[55] Colon, Y. J.; Snurr, R. Q. Chem. Soc. Rev. 2014, 43, 5735.

[56] Kong, X. Q.; Deng, H. X.; Yan, F. Y.; Kim, J.; Swisher, J. A.; Smit, B.; Yaghi, O. M.; Reimer, J. A. Science 2013, 341, 882.

[57] Tong, M.; Lan, Y. S.; Yang, Q. Y.; Zhong, C. L. Green Energy Environ. 2018, 3, 107.

[58] Deem, M. W.; Pophale, R.; Cheeseman, P. A.; Earl, D. J. J. Phys. Chem. C 2009, 113, 21353.

[59] Pophale, R.; Cheeseman, P. A.; Deem, M. W. Phys. Chem. Chem. Phys. 2011, 13, 12407.

[60] Bouëssel du Bourg, L.; Ortiz, A. U.; Boutin, A.; Coudert, F.-X. APL Mater. 2014, 2, 124110.

[61] Edgar, M.; Mitchell, R.; Slawin, A. M. Z.; Lightfoot, P.; Wright, P. A. Chem. Eur. J. 2001, 7, 5168 .

[62] Tian, C. B.; Chen, R. P.; He, C.; Li, W. J.; Wei, Q.; Zhang, X. D.; Du, S. W. Chem. Commun. (Camb.) 2014, 50, 1915.

[63] Sikora, B. J.; Wilmer, C. E.; Greenfield, M. L.; Snurr, R. Q. Chem. Sci. 2012, 3, 2217.

[64] Erucar, I.; Keskin, S. Front. Mater. 2018, 5, 4.

[65] Sarkisov, L.; Harrison, A. Mol. Simul. 2011, 37, 1248.

[66] First, E. L.; Gounaris, C. E.; Wei, J.; Floudas, C. A. Phys. Chem Chem. Phys. 2011, 13, 17339.

[67] Alexandrov, E. V.; Blatov, V. A.; Kochetkov, A. V.; Proserpio, D. M. CrystEngComm 2011, 13, 3947.

[68] Becker, T. M.; Heinen, J.; Dubbeldam, D.; Lin, L. C.; Vugt, T. J. H. J. Phys. Chem. C 2017, 121, 4659.

[69] McDaniel, J. G.; Schmidt, J. R. J. Phys. Chem. C 2012, 116, 14031.

[70] Mercado, R.; Vlaisayljevich, B.; Lin, L. C.; Lee, K.; Lee, Y.; Mason, 
J. A.; Xiao, D. J.; Gonzalez, M. I.; Kapelewski, M. T.; Neaton, J. B.; Smit, B. J. Phys. Chem. C 2016, 120, 12590.

[71] Rappé, A. K.; Casewit, C. J.; Colwell, K. S.; Goddard III, W. A.; Skiff, W. M. J. Am. Chem. Soc. 1992, 114.25, 10024.

[72] Mayo, S. L.; Olafson, B. D.; Goddard, W. A. J. Phys. Chem. 1990, 94, 8897.

[73] Grajciar, L.; Nachtigall, P.; Bludský, O.; Rubeš, M. J. Chem. Theory Comput. 2014, 11, 230.

[74] Rappe, A. K.; Goddard III, W. A. J. Phys. Chem. 1991, 95, 3358.

[75] Wilmer, C. E.; Kim, K. C.; Snurr, R. Q. J. Phys. Chem. Lett. 2012, 3, 2506.

[76] Xu, Q.; Zhong, C. L. J. Phys. Chem. C 2010, 114, 5035.

[77] Kadantsev, E. S.; Boyd, P. G.; Daff, T. D.; Woo, T. K. J. Phys. Chem. Lett. 2013, 4, 3056.

[78] Li, S.; Chung, Y. G.; Snurr, R. Q. Langmuir 2016, 32, 10368.

[79] Li, W.; Rao, Z. Z.; Chung, Y. G.; Li, S. ChemistrySelect 2017, 2, 9458.

[80] Kresse, G.; Furthmüller, J. Comput. Mater. Sci. 1996, 6, 15.

[81] Kresse, G.; Furthmüller, J. Phys. Rev. B 1996, 54, 11169.

[82] Kresse, G.; Hafner, J. Phys. Rev. B 1993, 47, 558.

[83] Kresse, G.; Hafner, J. Phys. Rev. B 1994, 49, 14251.

[84] Campaná, C.; Mussard, B.; Woo, T. K. J. Chem. Theory Comput. 2009, $5,2866$.

[85] Manz, T. A.; Sholl, D. S. J. Chem. Theory Comput. 2012, 8, 2844.

[86] Nazarian, D.; Camp, J. S.; Chung, Y. G.; Snurr, R. Q.; Sholl, D. S. Chem. Mater. 2016, 29, 2521.

[87] Hirschfelder, J. O.; Curtiss, C. F.; Bird, R. B.; Mayer, M. G. Molecular Theory of Gases and Liquids, Wiley, New York, 1954.

[88] Talu, O.; Myers, A. L. Colloids Surf. A 2001, 187, 83.

[89] Yang, Q. Y.; Zhong, C. L. J. Phys. Chem. B 2005, 109, 11862.

[90] Wilmer, C. E.; Farha, O. K.; Bae, Y. S.; Hupp, J. T.; Snurr, R. Q. Energy Environ. Sci. 2012, 5, 9849.

[91] Fernandez, M.; Woo, T. K.; Wilmer, C. E.; Snurr, R. Q. J. Phys. Chem. C 2013, 117, 7681.

[92] Michels, A.; De Graaff, W.; Ten Seldam, C. A. Physica 1960, 26, 393.

[93] Lamari, F. D.; Levesque, D. J. Chem. Phys. 1998, 109, 4981.

[94] Gomez, D. A.; Toda, J.; Sastre, G. Phys. Chem. Chem. Phys. 2014, 16, 19001.

[95] Zhang, H. D.; Deria, P.; Farha, O. K.; Hupp, J. T.; Snurr, R. Q. Energy Environ. Sci. 2015, 8, 1501.

[96] Liu, Y.; Guo, F. Y.; Hu, J.; Zhao, S. L.; Liu, H. L.; Hu, Y. Chem. Eng. Sci. 2015, 137, 170.

[97] Buch, V.; Devlin, J. P. J. Chem. Phys. 1993, 98, 4195.

[98] Guo, F. Y.; Liu, Y.; Hu, J.; Liu, H. L.; Hu, Y. Chem. Eng. Sci. 2016, 149, 14.

[99] Li, S.; Chung, Y. G.; Snurr, R. Q. Langmuir 2016, 32, 10368.

[100] Moghadam, P. Z.; Fairen-Jimenez, D.; Snurr, R. Q. J. Mater. Chem. A 2016, 4, 529.

[101] Nazarian, D.; Camp, J. S.; Sholl, D. S. Chem. Mater. 2016, 28, 785.

[102] Qiao, Z. W.; Peng, C. W.; Zhou, J.; Jiang, J. W. J. Mater. Chem. A 2016, 4, 15904.

[103] Altintas, C.; Keskin, S. Chem. Eng. Sci. 2016, 139, 49.

[104] Erucar, I.; Keskin, S. J. Membr. Sci. 2016, 514, 313.

[105] Aghaji, M. Z.; Fernandez, M.; Boyd, P. G.; Daff, T. D.; Woo, T. K. Eur. J. Inorg. Chem. 2016, 2016, 4505.

[106] Fernandez, M.; Barnard, A. S. ACS Comb. Sci. 2016, 18, 243.

[107] Chung, Y. G.; Gomez-Gualdron, D. A.; Li, P.; Leperi, K. T.; Deria, P.; Zhang, H. D.; Vermeulen, N. A.; Stoddart, J. F.; You, F. Q.; Hupp, J. T.; Farha, O. K.; Snurr, R. Q. Sci. Adv. 2016, 2, e1600909.

[108] Chung, Y. G.; Bai, P.; Haranczyk, M.; Leperi, K. T.; Li, P.; Zhang, H. D.; Wang, T. C.; Duerinck, T.; You, F. Q.; Hupp, J. T.; Farha, O. K.; Siepmann, J. I.; Snurr, R. Q. Chem. Mater. 2017, 29, 6315.
[109] Boato, G.; Casanova, G. Physica 1961, 27, 571.

[110] Van Heest, T.; Teich-McGoldrick, S. L.; Greathouse, J. A.; Allendorf, M. D.; Sholl, D. S. J. Phys. Chem. C 2012, 116, 13183.

[111] Pardakhti, M.; Moharreri, E.; Wanik, D.; Suib, S. L.; Srivastava, R. ACS Comb. Sci. 2017, 19, 640.

[112] Borboudakis, G.; Stergiannakos, T.; Frysali, M.; Klontzas, E.; Tsamardinos, I.; Froudakis, G. E. npj Comput. Mater. 2017, 3, 1.

[113] Kadioglu, O.; Keskin, S. Sep. Purif. Technol. 2018, 191, 192.

[114] Martin, M. G.; Siepmann, J. I. J. Phys. Chem. B 1998, 102, 2569.

[115] Buch, V. J. Chem. Phys. 1994, 100, 7610.

[116] Altintas, C.; Erucar, I.; Keskin, S. ACS Appl. Mater. Interfaces 2018 10,3668 .

[117] Budhathoki, S.; Ajayi, O.; Steckel, J. A.; Wilmer, C. E. Energy Environ. Sci. 2018, DOI: 10.1039/c8ee02582g.

[118] Qiao, Z. W.; Xu, Q. S.; Jiang, J. W. J. Membr. Sci. 2018, 551, 47.

[119] Anderson, R.; Rodgers, J.; Argueta, E.; Biong, A.; Gómez-Gualdrón, D. A. Chem. Mater. 2018, 30, 6325.

[120] Potoff, J. J.; Siepmann, J. I. AIChE J. 2001, 47, 1676.

[121] Serratosa, J. M.; Gómez-Garre, P.; Gallardo, M. E.; Anta, B.; De Bernabé, D. B.-V.; Lindhout, D.; Augustijn, P. B.; Tassinari, C. A.; Michelucci, R.; Malafosse, A. Hum. Mol. Genet. 1999, 8, 345.

[122] Jorgensen, W. L.; Chandrasekhar, J.; Madura, J. D.; Impey, R. W.; Klein, M. L. J. Chem. Phys. 1983, 79, 926.

[123] Horn, H. W.; Swope, W. C.; Pitera, J. W.; Madura, J. D.; Dick, T. J.; Hura, G. L.; Head-Gordon, T. J. Chem. Phys. 2004, 120, 9665.

[124] Raccuglia, P.; Elbert, K. C.; Adler, P. D.; Falk, C.; Wenny, M. B.; Mollo, A.; Zeller, M.; Friedler, S. A.; Schrier, J.; Norquist, A. J. Nature 2016, 533, 73 .

[125] Schalkoff, R. J. Artificial Neural Networks, McGraw-Hill, New York, 1997.

[126] Gandara, F.; Furukawa, H.; Lee, S.; Yaghi, O. M. J. Am. Chem. Soc. 2014, 136, 5271.

[127] Koh, H. S.; Rana, M. K.; Wong-Foy, A. G.; Siegel, D. J. J. Phys Chem. C 2015, 119, 13451.

[128] Wang, X.; Fordham, S.; Zhou, H. C. ACS Symp. Ser. 2015, 1213, 173 .

[129] Zhang, H.; Li, G. L.; Zhang, K. G.; Liao, C. Y. Acta Chim. Sinica 2017, 75, 841. (张贺, 李国良, 张可刚, 廖春阳, 化学学报, 2017, 75,841 .)

[130] DOE targets for onboard hydrogen storage systems for light-duty vehicles, http://www1.eere.energy.gov/hydrogenandfuelcells/ storage/pdfs/targets onboard hydro storage.pdf.

[131] The Toyota Fuel Cell Vehicle: a turning point from the inside out, http://www.toyota.com/mirai/fcv.html.

[132] Total hydrogen station in Munich first to feature standard compressed $\mathrm{H} 2$ and BMW cryocompressed $\mathrm{H} 2$ technology, http://www. greencardcongress.com/2015/07/20150715.

[133] Engineering an adsorbent based hydrogen storage system: What have we learned? https://www.energy.gov/sites/prod/files/2015/02/ f19/fcto h2 storage summit siegel.pdf.

[134] Kale, C.; Gorak, A.; Schoenmakers, H. Int. J. Greenhouse Gas Control 2013, 17, 294.

[135] Jameson, C. J.; Jameson, A. K.; Lim, H. M. J. Chem. Phys. 1997, 107, 4364.

[136] Ryan, P.; Farha, O. K.; Broadbelt, L. J.; Snurr, R. Q. AIChE J. 2011, $57,1759$.

[137] Wu, L. M.; Xiao, J.; Wu, Y.; Xian, S. K.; Miao, G.; Wang, H. H.; Li, Z. Langmuir 2014, 30, 1080.

[138] Bian, L.; Li, W.; Wei, Z. Z.; Liu, X. W.; Li, S. Acta Chim. Sinica 2018, 76, 303. (市磊, 李炜, 魏振振, 刘晓威, 李松, 化学学报, 2018, 76, 303.) 